NISTIR 8177

\title{
Metrics and Key Performance Indicators for Robotic Cybersecurity Performance Analysis
}

Timothy A. Zimmerman

This publication is available free of charge from:

https://doi.org/10.6028/NIST.IR.8177

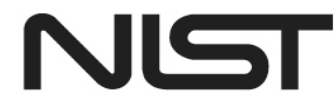

National Institute of Standards and Technology U.S. Department of Commerce 


\section{Metrics and Key Performance Indicators for Robotic Cybersecurity Performance Analysis}

Timothy A. Zimmerman

Intelligent Systems Division

Engineering Laboratory

This publication is available free of charge from:

https://doi.org/10.6028/NIST.IR.8177

March 2017

INCLUDES UPDATES AS OF May 21, 2019

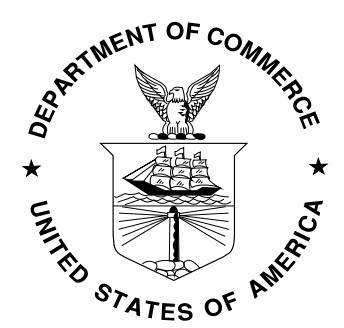

U.S. Department of Commerce Wilbur L. Ross, Jr., Secretary

National Institute of Standards and Technology Kent Rochford, Acting NIST Director and Under Secretary of Commerce for Standards and Technology 


\section{CONTENTS}

Contents . i

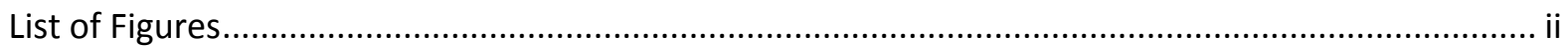

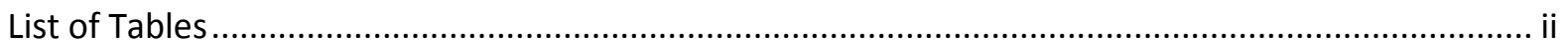

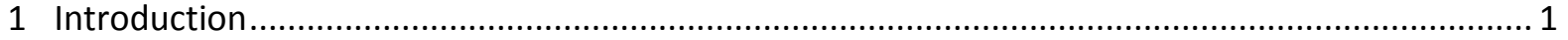

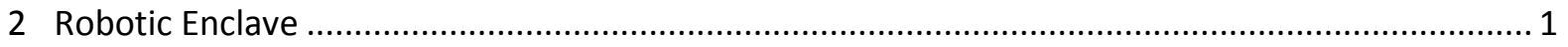

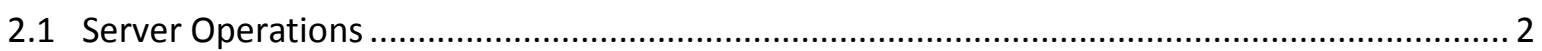

2.2 Emulated Manufacturing Operation..................................................................................... 3

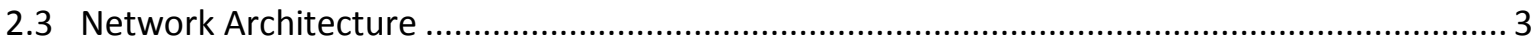

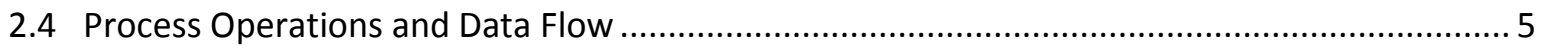

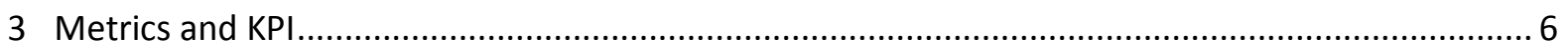

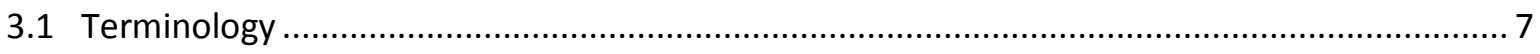

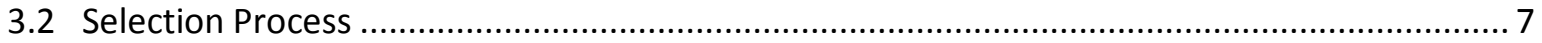

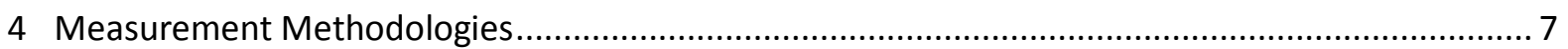

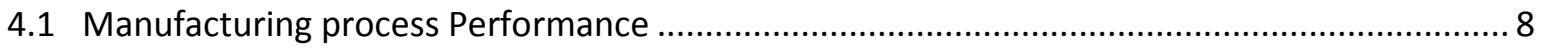

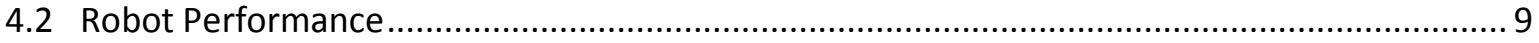

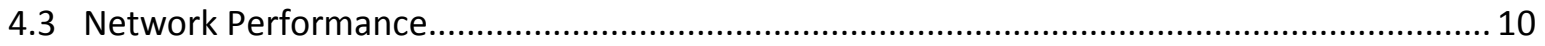

4.4 Programmable Logic Controller Performance ................................................................... 11

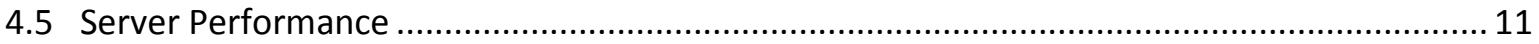

5 Appendix A - Description of Measurements and KPI.............................................................. 13

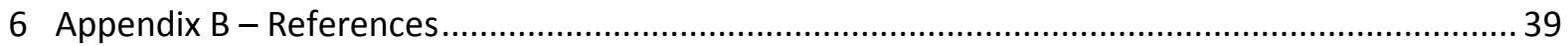




\section{LIST OF FIGURES}

Figure 1 - View of the robotic enclave and its components......................................................... 2

Figure 2 - Flow chart of the emulated manufacturing process .......................................................... 3

Figure 3 - Diagram of the network architecture and its components............................................ 4

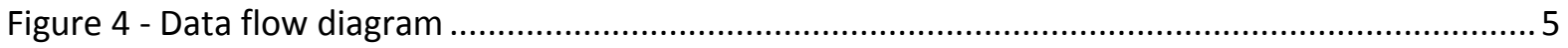

\section{LIST OF TABLES}

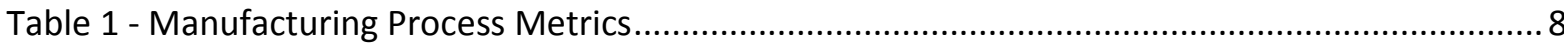

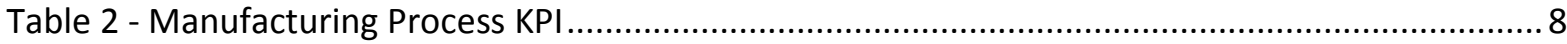

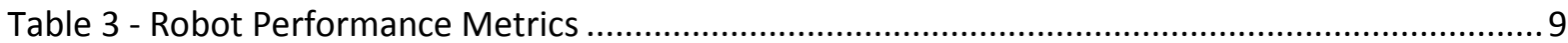

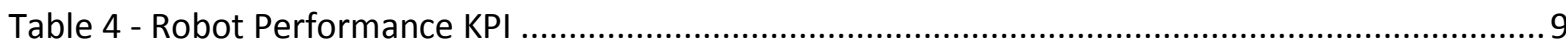

Table 5 - Network Performance Metrics …….............................................................................. 10

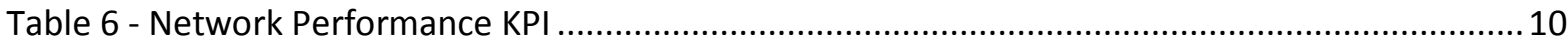

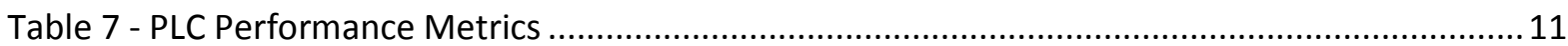

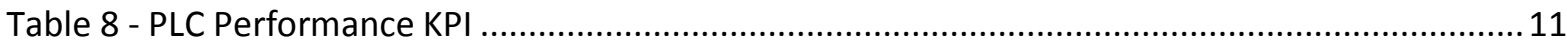

Table 9 - Server Performance Metrics......................................................................................... 12

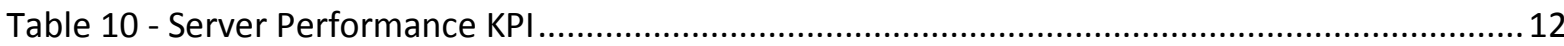

\section{Disclaimer}

Certain commercial entities, equipment, or materials may be identified in this document in order to describe an experimental procedure or concept adequately. Such identification is not intended to imply recommendation or endorsement by NIST, nor is it intended to imply that the entities, materials, or equipment are necessarily the best available for the purpose. 


\section{ERRATA}

This table contains changes that have been incorporated into NISTIR 8177. Errata updates can include corrections, clarifications, or other minor changes in the publication that are either editorial or substantive in nature.

\begin{tabular}{|c|c|l|c|}
\hline Date & \multicolumn{1}{|c|}{ Type } & \multicolumn{1}{|c|}{ Change } & Page \\
\hline 04-24-2019 & Editorial & General formatting. & All \\
\hline 04-24-2019 & Substantive & Updated description for KPI 4.1 (Actuation Latency) in Table 4. & 9 \\
\hline $04-24-2019$ & Substantive & Added new KPI 4.6 (Job Execution Time) in Table 4. & 9 \\
\hline 04-24-2019 & Substantive & $\begin{array}{l}\text { Incorporated the original notes for Throughput Rate (KPI 2.3) into } \\
\text { the general formulas and fixed a mathematical error. }\end{array}$ & 15 \\
\hline 04-24-2019 & Substantive & Fixed variable terms for KPI 3.2 (Joint Setpoint). & 18 \\
\hline 04-24-2019 & Substantive & $\begin{array}{l}\text { Updated the description, formula, and notes for KPI 4.1 } \\
\text { (Actuation Latency). }\end{array}$ & 19 \\
\hline 04-24-2019 & Substantive & Updated the formula and notes for KPI 4.2 (Pose Travel Time). & 20 \\
\hline 04-24-2019 & Editorial & Restructured formula layout for KPI 4.3 (Position Accuracy). & 20 \\
\hline 04-24-2019 & Substantive & Added new KPI 4.6 (Job Execution Time). & 22 \\
\hline 04-24-2019 & Substantive & $\begin{array}{l}\text { Added bar accent to formula for KPI 6.6 to denote the calculation } \\
\text { produces an average value. }\end{array}$ & 26 \\
\hline 04-24-2019 & Substantive & $\begin{array}{l}\text { Added bar accent to formula for KPI 6.7 to denote the calculation } \\
\text { produces an average value. }\end{array}$ & 27 \\
\hline 04-24-2019 & Substantive & Fixed error in formula for KPI 8.2 (CPU Utilization Distribution). & 29 \\
\hline 04-24-2019 & Editorial & Fixed formatting error, separating tables for KPI 8.2 and KPI 9.1. & 30 \\
\hline
\end{tabular}




\section{INTRODUCTION}

The National Institute of Standards and Technology has constructed a testbed to measure the performance impact induced by cybersecurity technologies on Industrial Control Systems (ICS) [1]. The testbed allows researchers to emulate real-world industrial manufacturing processes and their control systems without the need to replicate an entire factory environment or its machinery.

The focus of this report is the Robotic Enclave of the testbed, which is comprised of two robotic arms that emulate a material handling application, known as "machine tending." Robotic machine tending uses robots to interact with the machinery, performing operations a human operator would normally perform (e.g., the loading and unloading of parts, opening and closing of machine doors, activating operator control panel buttons). In the enclave, parts are transported collaboratively through simulated sequential machining operations, known as "stations." The enclave was designed and constructed to be reconfigurable, allowing numerous types of operational methodologies, network topologies, and industrial networking protocols to be investigated.

The research performed on the enclave will explore the effects of implementing cybersecurity technologies, as defined by industry best-practices and standards, on the testbed operation and measure the performance impact. As later sections will elaborate, the impact will be measured simultaneously across many of the enclave subsystems during an experiment to provide a holistic understanding of the underlying effects of the defenses.

This report identifies the metrics, Key Performance Indicators (KPI), their derivations, and the measurement methodologies that will be employed during future enclave experiments.

\section{ROBOTIC ENCLAVE}

The Robotic Enclave, shown in Figure 1, is one of four enclaves within the Cybersecurity for Smart Manufacturing Systems (CSMS) testbed. It includes two robotic arms that emulate a machine tending application, where parts are loaded, unloaded, and transported between sequential machining operations in a simulated batch-production process.

The robots operate in concert according to a material handling procedure that changes dynamically based on feedback from the simulated machining operations. In addition to the two industrial robots, the enclave includes a supervisory programmable logic controller (PLC), a safety PLC, a human machine interface (HMI), a real-time vision tracking system, several servers for executing required computational resources and applications, and an engineering workstation. 
Figure 1 - View of the robotic enclave and its components

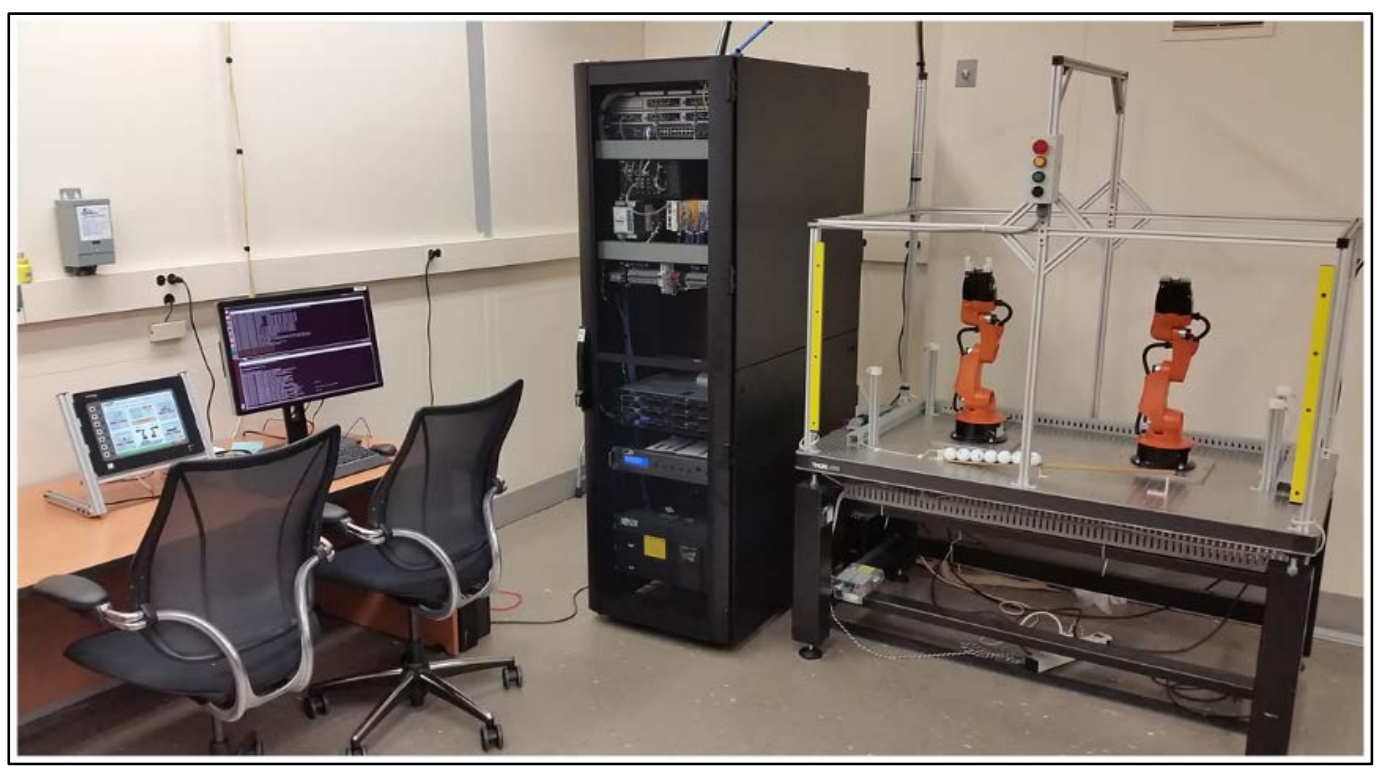

\subsection{SERVER OPERATIONS}

The robot controllers can operate in one of two modes: deployed or virtualized. In the deployed mode, each robot is controlled on a dedicated Dell PowerEdge R420 server running the Robot Operating System (ROS) on top of Ubuntu Linux. In the virtualized mode, each robot is controlled by virtualized servers within a hypervisor running on a Dell PowerEdge 620 server. The deployed mode supports experiments with a pseudo-ideal configuration, and the virtualized mode supports experiments with a resource-restricted configuration, as well as the ability to maintain independent testing environments.

The pseudo-ideal configuration provides the robot controller software with computational resources well-beyond the minimum requirements for unimpeded operations. Operating in this manner is reserved for experiments that do not require server performance impacts to be measured (e.g., network-specific experiments). The resource-restricted configuration allows the researchers to restrict the available resources to the robot controller software and underlying operating system (e.g., memory allocation, available hard disk space, hard disk access rates, number of central processing unit (CPU) cores).

The hypervisor also allows software-based cybersecurity tools to be deployed within an isolated environment for testing and the ability to restore the enclave environment to a known-good state, reducing the chances of cross-contamination by residual software modules or services remaining within a virtual machine post-experiment. Software-based cybersecurity tools are installed on virtual machines dedicated to specific experiments within the hypervisor, and archived. This allows any tool to be recalled for any experiment that requires its execution. 


\subsection{EMULATED MANUFACTURING OPERATION}

The sequential machining operations emulated by the enclave are shown in Figure 2 . The robots work collaboratively to move the parts between the successive stations. Each station is physically constructed from an additive manufactured base with a concave recess to capture the parts, and an integrated proximity sensor. The parts transported between the stations are acetal resin spheres, $38 \mathrm{~mm}$ (1.5 inch) in diameter.

Figure 2 - Flow chart of the emulated manufacturing process

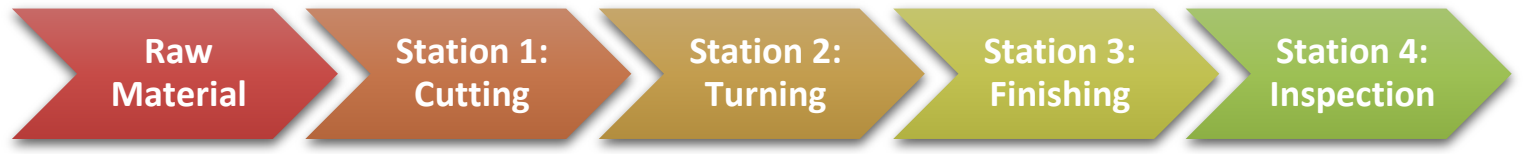

The machining operations of each station are emulated as configurable timers within the supervisory PLC (Beckhoff CX9020) with three operational states: "idle," "processing," and "ready for pickup." A station is in the "idle" state when no part is present, the "processing" state when a part is present and the timer has not reached its set duration, and the "ready for pickup" state when a part is present and the timer has reached its set duration. The status information for each machining station is made available to other network devices (e.g., robot controllers, HMI) via a Modbus TCP server on the PLC.

\subsection{NETWORK ARCHITECTURE}

The enclave local area network (ICS LAN) is constructed as a flat architecture, as shown in Figure 3. For the initial experimentation, the architecture will remain flat (as this is most prevalent in industry). However, the reconfigurable design of the enclave will enable the implementation of network segmentation and security perimeters during future experiments. The local network traffic ("ICS LAN") is managed by a Siemens RUGGEDCOM RX1510, and the high-level testbed traffic ("Testbed LAN") and its connection to the "Corporate Network" are managed by a Cisco ASA 5512-X.

The Testbed LAN has three machines: a probe server, source control management (SCM) server, and Network Time Protocol (NTP) and Precision Time Protocol (PTP) capable master clock. The probe server ("ORION") consumes and records network traffic from twelve independent network interfaces. The SCM server ("RIGEL") runs a GitLab server to track and maintain the code used on the enclave, primarily robot code, PLC code, and analysis tools. The NTP/PTP clock ("GEORGE") provides stable time to the enclave servers and systems to enable synchronized logging.

The ICS LAN has numerous machines that directly operate and support the operation of the enclave. The robot controller/driver servers ("MINTAKA", "SAIPH") execute the operational code and communicate directly with the robots to direct their actions. The supervisory PLC ("GIZMO") communicates the status of the machining stations and operator controls to the robot controllers, as well as part tracking for manufacturing performance measurements. The operator $\mathrm{HMI}$ also communicates with the PLC to display manufacturing process information and performance measurements to the operator. The engineering workstation ("POLARIS") hosts the programming environment and debugging tools used to modify the robot code, and give terminal-level access to other machines within the 
enclave. The HyperV server ("MEISSA") provides server virtualization to the enclave, allowing researchers to create servers on-demand, as required by specific software tools or packages.

Network probes within the enclave intercept network traffic at key points and mirror the traffic to the measurement enclave via a patch panel. Within the measurement enclave, packets can be captured, manipulated, and logged using a collection of network testing hardware and software.

Figure 3 - Diagram of the network architecture and its components

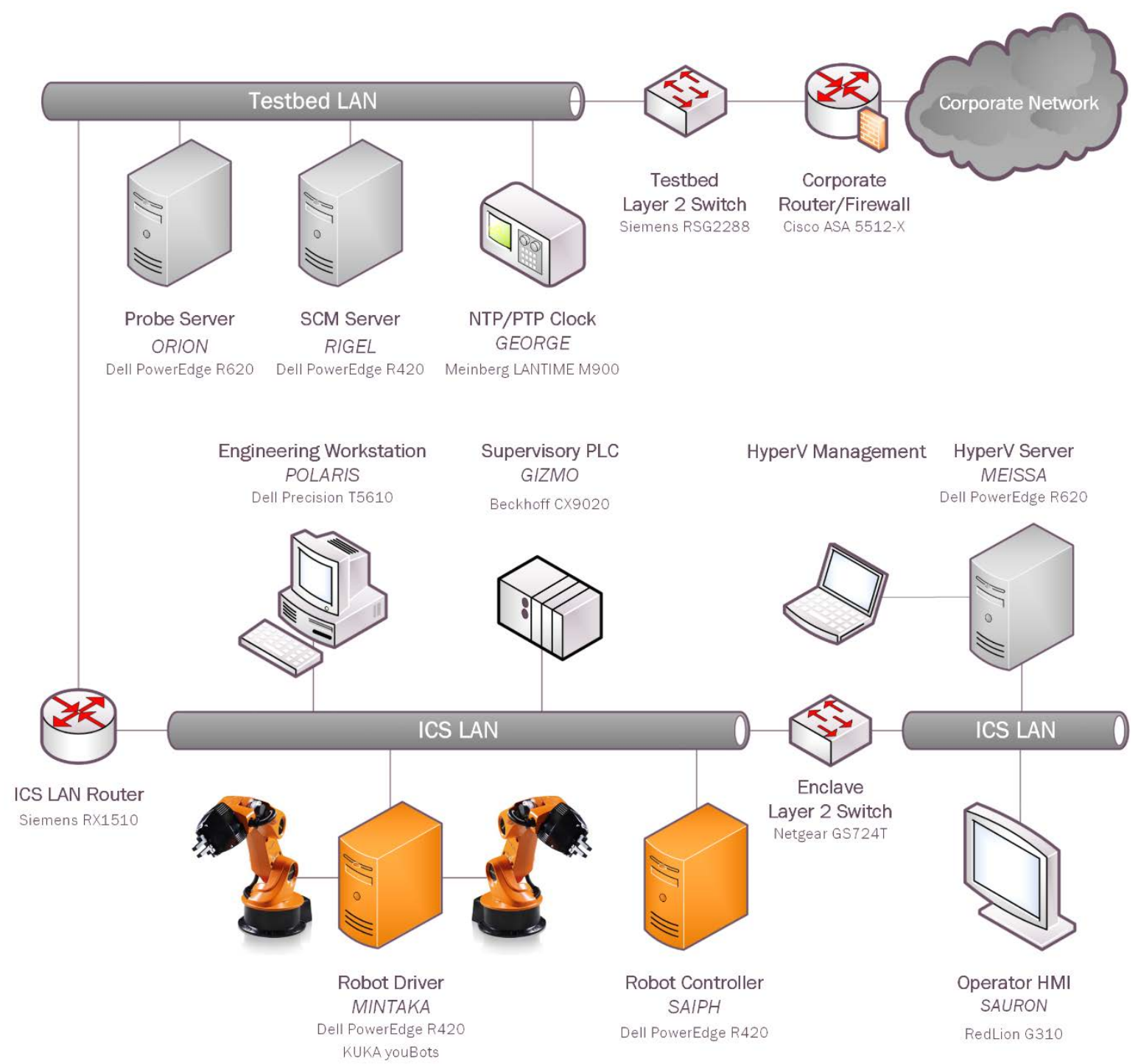




\subsection{PROCESS OPERATIONS AND DATA FLOW}

Figure 4 illustrates the data flow of the processes and components within the enclave. All operations are initiated by the PLC, which monitors the operator buttons, $\mathrm{HMI}$, and safety systems. The robots will commence their material handling operations only when the PLC informs the robot controllers that it is safe to do so. This communication is enabled by a Modbus TCP server in the PLC, which the robot controllers can access directly via Modbus TCP polling. The Modbus server provides the controllers with the current operating state of the enclave, the status of each operator button and switch, and the status of each machining station.

Figure 4 - Data flow diagram

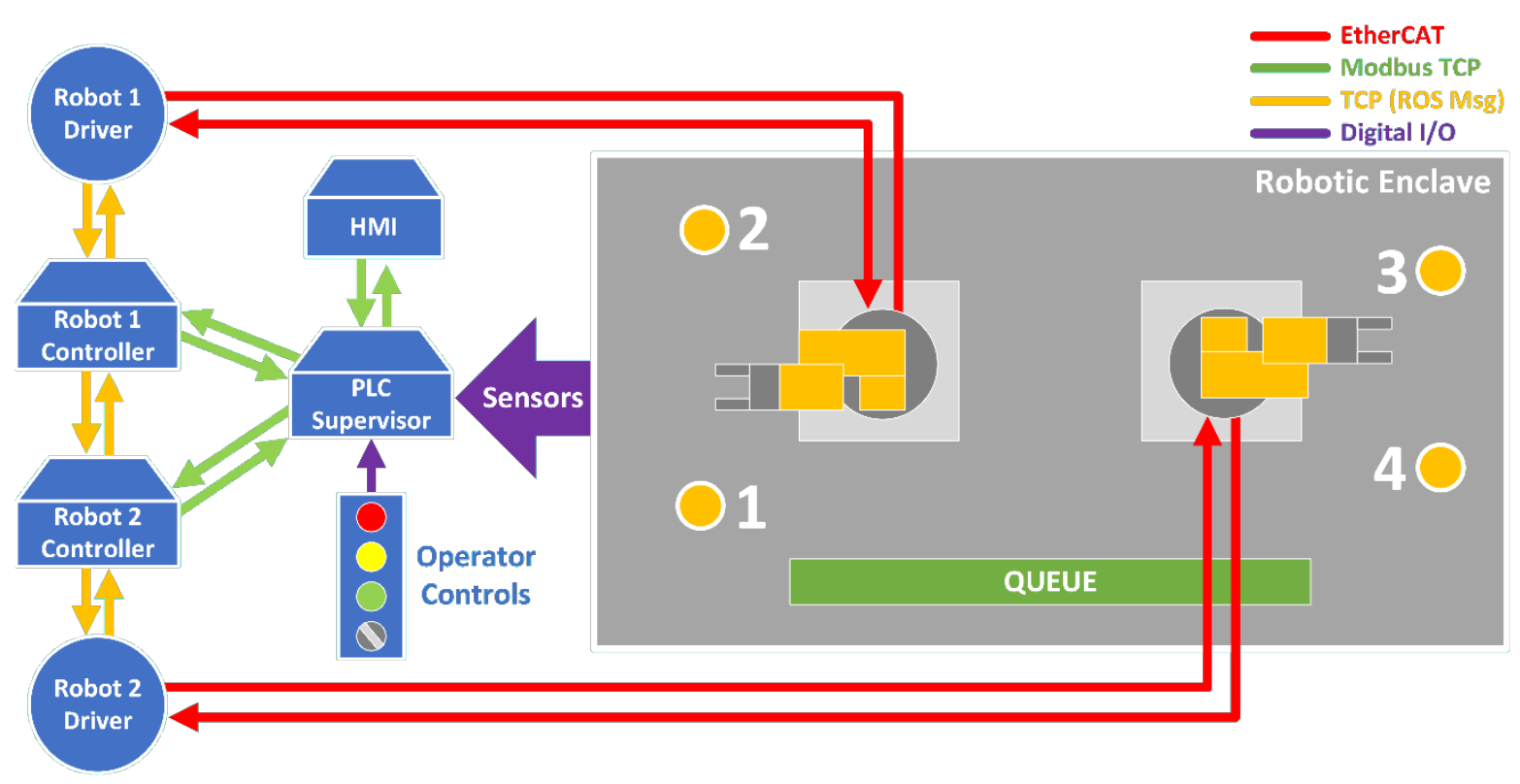

While a robot is idle, the controller will continue polling the Modbus Service to obtain the operating status of each machining station. This status information is passed through decision logic within the robot controller to determine what actions the robot should perform, based on the given machining station states. Once the controller logic determines which action should be executed, the arm will move through a series of predetermined poses and actions to complete the intended operation.

The predetermined poses and actions are described in a Yet Another Markup Language (YAML) file, which defines each discrete joint position or action (e.g., open/close the jaw) the arm should sequentially execute to complete the movement. For example, if Station \#1 reports a part is ready for pickup, Robot \#1's controller will check to see if there is a part at Station \#2. If Station \#2 is empty, the robot controller will execute the required set of actions to move the part from Station \#1 to Station \#2. The controller will continue executing each discrete action within the set until it has completed. At this point, the controller will return the robot to its idle position and continue scanning the machining 
stations until a new event triggers another movement to be performed (e.g., move a part from the raw material queue to the now empty Station \#1).

Parts will continue moving through the machining stations as long as there is raw material on the queue, and the experiment termination triggers within the $\mathrm{HMI}$ are not achieved. Experiment termination triggers available can be either part-counter based, or timer-based. Once a trigger is activated, the enclave will enter the "stop" state and allow the robots to complete their current movements before ceasing all operations.

The control of each robot joint is performed with a cascaded control architecture. Closed-loop proportional-integral-derivative (PID) position control is performed by the motor controllers for each joint. The enclave also has the capability to control the robot joints in a velocity control mode, where the PID position control is performed by the robot controllers, and velocity updates are sent over the network.

Because of the reconfigurable nature of the enclave, the hosts of the robot controller processes are excluded from Figure 4. However, in the deployed mode, the robot controller processes typically execute on SAIPH, with the driver processes executing on MINTAKA.

\section{METRICS AND KPI}

The research and selection of enclave metrics and KPI were driven by the experimental goal: to capture empirical evidence of performance impacts induced by cybersecurity protections (e.g., hardware, software, implementation of industry best-practices). Measurements of the manufacturing operation are captured and compared to those captured during baseline experiments. Statistical analysis will provide a method of detecting deviations from the expected behavior of the manufacturing process, although it is not the focus of this report.

The deviation of a specific measurement from the baseline will not directly identify the underlying cause. To resolve this, measurements from the subsystems that support the manufacturing operation are also captured. Simultaneously measuring at the manufacturing-level and subsystem-level enables analysis of the performance impact to the manufacturing operation, as well as the underlying subsystems and their interactions. In the robotic enclave, the high-level system is the discrete manufacturing operation, and supporting the operation are the enclave subsystems (e.g., servers, PLC, HMI, network equipment).

For an example of the interactions between the manufacturing process and underlying subsystems, consider a hypothetical manufacturing operation where an operator must regularly inspect the output product and manipulate machine variables via an HMI to keep the parts free of defects. The proposed cybersecurity protection to be implemented is "requiring a password on the HMI to prevent unauthorized users from manipulating the machine variables." If the machine in question produces products at a rate of 10 parts per hour, and it takes the operator an extra 5 seconds to unlock the $\mathrm{HMI}$, the performance impact to the manufacturing process will be negligible. However, if the machine operates at high-speeds, with production rates measured in parts per second, requiring the 
operator to regularly spend an additional 5 seconds to unlock the $\mathrm{HMI}$ and adjust the machine variables will cause performance impacts to the output production rate.

\subsection{TERMINOLOGY}

This report uses a naming convention that follows terminology to enable effective communications between researchers and industry, as proposed in [2]. More specifically, this report defines a metric as a "directly measurable property of a system," while a KPI is defined as a "computable performance assessment, as derived from a combination of metrics." Metrics are analogous to primitives, while KPIs are a form of post-analysis which utilize the primitives to compute an inference. For comparison, ISO 22400-1:2014 defines the measurements used in the calculation of KPIs as "elements."

Using the robotic enclave as an example, consider the following two metrics: the amount of time a single machining station is performing work on parts during a batch, and the total amount of time required to produce the batch. Both are directly measurable, and are considered metrics. However, effective communication of either metric proves problematic when comparing to systems with differing dynamics (i.e., an enclave with four machining stations compared to six machining stations). This is resolved by combining individual metrics within a computation to calculate a $\mathrm{KPI}$. Continuing with the previous example, the machining station working time and total batch production time can be used to calculate the "Utilization Efficiency" of the station (see KPI 2.4 in Section 4.1), which can be directly communicated and compared to other systems.

\subsection{SELECTION PROCESS}

The manufacturing process and various subsystems operating within the enclave were examined to determine which metrics could be obtained. Literature related to the performance of manufacturing systems and other industry standards were then reviewed to determine which KPI could be used to best analyze the manufacturing process with the available metrics. The literature in [3], [4], [5], [6], and [7] proved most helpful by providing insight into some of the more elusive KPI for the testbed subsystems. KPIs that did not provide results related to the research goal were not considered.

\section{MEASUREMENT METHODOLOGIES}

To detect and measure performance impacts to the manufacturing system, specific metrics from the manufacturing process, robots, local network, PLC, and servers are captured. These metrics are then used to calculate KPIs to provide quantifiable and communicable indicators, and detect any performance impacts. If any impacts are detected, more detailed analysis can be performed to assist with locating the source of the performance degradation.

The following sections describe the measurement methodology used for each process and subsystem, as well a summary of the metrics and KPIs. Detailed descriptions and formulas for each metric and KPI are located in Appendix A - Description of Measurements and KPI. 


\subsection{MANUFACTURING PROCESS PERFORMANCE}

For each batch produced, the PLC captures all the metrics listed in Table 1. Arrival and departure timestamps for each station are stored in a multidimensional array, and the station counters are implemented as primitive variables. When the production process is started, the PLC will log the start time obtained from the grandmaster NTP clock (labeled GEORGE in Figure 3). Once the experiment start time is logged, the PLC enables enclave operations: Modbus server registers are updated to inform all subsystems that operations are enabled, the counters begin incrementing, and the recording of each station event is enabled.

Counters and event trackers are updated on every cycle of the PLC task, which is $10 \mathrm{~ms}$ at the time of writing. When a part enters or exits a station, a timestamp of the event is stored in the part tracking array, relative to the start of the batch. The PLC also captures the total amount of time each station spends in any valid state. The three valid states for a machining station are: busy, waiting, and idle. The busy state represents the time a station is actively machining a part. After machining has completed, the station enters the waiting state, and waits for a robot to remove the part. Once the part is removed from the station, it enters the idle state until a new part is loaded. Batch production time and the total number of parts produced are also tracked, as they are required for some of the KPI calculations.

When the enclave completes its production cycle, all process data remains within the PLC until it is downloaded and purged by an operator via the HMI. The KPIs, listed in Table 2, can be computed in real-time via the operator $\mathrm{HMI}$, or offline after the experiment has been completed.

Table 1 - Manufacturing Process Metrics

\begin{tabular}{|l|l|}
\hline \multicolumn{1}{|c|}{ Metric } & \multicolumn{1}{c|}{ Description } \\
\hline 1.1 Part Timestamps & $\begin{array}{l}\text { Timestamping of unique part events (e.g., arriving and de- } \\
\text { parting stations) }\end{array}$ \\
\hline 1.2 Station Timers & Accumulation of time a station is in any valid state \\
\hline 1.3 Batch Timer & $\begin{array}{l}\text { Total amount of time required for the batch to be pro- } \\
\text { duced }\end{array}$ \\
\hline 1.4 Batch Parts Counter & Number of parts produced in the batch \\
\hline
\end{tabular}

Table 2 - Manufacturing Process KPI

\begin{tabular}{|l|l|}
\hline \multicolumn{1}{|c|}{ Key Performance Indicator } & \multicolumn{1}{c|}{ Description } \\
\hline 2.1 Part Production Time & Amount of time required for a part to be produced \\
\hline 2.2 Cycle Time & Amount of time between finished parts \\
\hline 2.3 Throughput Rate & Number of parts produced over a specific amount of time \\
\hline 2.4 Production Effectiveness & $\begin{array}{l}\text { Relationship between the planned production time to com- } \\
\text { plete a batch and the actual production time }\end{array}$ \\
\hline 2.5 Station Allocation Ratio & $\begin{array}{l}\text { Ratio between the amount of time a station was busy and } \\
\text { the batch production time }\end{array}$ \\
\hline
\end{tabular}




\subsection{Station Utilization Efficiency}

Ratio between the amount of time a station was producing and the amount of time the batch required to complete

\subsection{ROBOT PERFORMANCE}

Robot performance metrics can be obtained by capturing the command and control communications used by the robots. Since the communication protocol used by the robots (EtherCAT) leverages existing Ethernet hardware, the packets can be captured directly from the physical interface, or with an in-line tool. Each packet contains all of the metrics shown in Table 3, and are transmitted at a rate of $700 \mathrm{~Hz}$. This communication is the lowest-level that can be captured, as packets on this path go directly to each individual joint of the robots.

Because of the huge amount of data being transmitted during a batch, capturing data on this interface is time-limited, and is typically reserved for experiments where robot performance impact is anticipated because of interim results obtained from more readily available metrics and KPIs.

When a capture of this interface is performed, all of the network traffic is logged through a Wireshark or tcpdump capture. The EtherCAT protocol data is then extracted from the packets offline by a custom Python dissector script.

Table 3 - Robot Performance Metrics

\begin{tabular}{|l|l|}
\hline \multicolumn{1}{|c|}{ Metric } & \multicolumn{1}{c|}{ Description } \\
\hline 3.1 Joint Position & Position of each robot joint \\
\hline 3.2 Joint Setpoint & Control setpoint of each robot joint \\
\hline 3.3 Joint Velocity & Velocity of each robot joint \\
\hline 3.4 Joint Current & Electrical current of each robot joint \\
\hline 3.5 Joint Control Flags & Flags describing the operational status of each robot joint \\
\hline
\end{tabular}

Table 4 - Robot Performance KPI

\begin{tabular}{|l|l|}
\hline \multicolumn{1}{|c|}{ Key Performance Indicator } & \multicolumn{1}{c|}{ Description } \\
\hline 4.1 Actuation Latency & $\begin{array}{l}\text { Amount of time required for the robot arm to begin move- } \\
\text { ment after a new job is received }\end{array}$ \\
\hline 4.2 Pose Travel Time & $\begin{array}{l}\text { Amount of time for the robot arm to move between two } \\
\text { poses }\end{array}$ \\
\hline 4.3 Position Accuracy & $\begin{array}{l}\text { Deviation between the commanded position and the } \\
\text { measured position }\end{array}$ \\
\hline 4.4 Position Repeatability & $\begin{array}{l}\text { Closeness of agreement between the measured position } \\
\text { after } n \text { repeat visits to the same commanded position }\end{array}$ \\
\hline 4.5 Energy Consumption & $\begin{array}{l}\text { Estimation of the amount of power consumed by the robot } \\
\text { joint motors }\end{array}$ \\
\hline 4.6 Job Execution Time & Amount of time required for a robot to complete a job \\
\hline
\end{tabular}




\subsection{NETWORK PERFORMANCE}

The network capture server within the measurement enclave is tasked with capturing all transmitted packets within the local network. Each packet is stored in its raw format, allowing numerous metrics to be obtained. Key nodes within the enclave have their Ethernet communications passed through an in-line Ethernet network tap (USRobotics USR4503). The packets are aggregated and mirrored to the measurement rack, where they are captured by a Dell PowerEdge R620 containing two Broadcom 5719 network interface cards, adding eight dedicated Ethernet packet capture interfaces.

A reconfigurable Python script and dissector were created to perform the capture and dissection processes. The script creates a dedicated tcpdump process for each Ethernet interface, as defined by the user in a configuration file, and stores captured packets in PCAP files. Dissection of ROS packets is performed using a Python script that contains the structures and signatures of known message types. The script can also automatically cease its measurements by listening for broadcast messages on the local network marking the end of an experiment.

Table 5 - Network Performance Metrics

\begin{tabular}{|ll|l|}
\hline \multicolumn{1}{|c|}{ Metric } & \multicolumn{1}{c|}{ Description } \\
\hline 5.1 & Packet Headers & Header data of a captured packet \\
\hline 5.2 & Packet Data & Application layer payload of a captured packet \\
\hline 5.3 Packet Protocol Type & Protocol type of a captured packet \\
\hline 5.4 Packet Timestamp & Timestamp of a captured packet \\
\hline 5.5 Packet Size & Size of a packet in bytes \\
\hline 5.6 & Packet Errors & Number of packets transmitted or received with errors \\
\hline 5.7 & Dropped Packets & Number of packets dropped by the network \\
\hline 5.8 Packet Counter & Number of packets transmitted and received \\
\hline
\end{tabular}

Table 6 - Network Performance KPI

\begin{tabular}{|ll|l|}
\hline \multicolumn{1}{|c|}{ Key Performance Indicator } & \multicolumn{1}{c|}{ Description } \\
\hline 6.1 & Packet Path Delay & Time delay along the path from transmitter to receiver \\
\hline 6.2 Inter-packet Delay & Difference between the packet path delay of two packets \\
\hline $6.3 \quad$ TCP Packet Round Trip Time & $\begin{array}{l}\text { Amount of time for the source node of a packet to receive } \\
\text { the acknowledgement of receipt (ACK) from the destina- } \\
\text { tion node }\end{array}$ \\
\hline 6.4 Information Ratio & $\begin{array}{l}\text { Ratio of the quantity of process information packets to all } \\
\text { packets }\end{array}$ \\
\hline 6.5 Bit Rate & $\begin{array}{l}\text { Rate of bits transmitted or received over a specific } \\
\text { timespan }\end{array}$ \\
\hline 6.6 Packet Rate & $\begin{array}{l}\text { Rate of packets transmitted and received over a specific } \\
\text { amount of time }\end{array}$ \\
\hline
\end{tabular}




\begin{tabular}{|l|l|}
\hline 6.7 Packet Error Rate & $\begin{array}{l}\text { Rate of packets transmitted or received with errors over a } \\
\text { specific amount of time }\end{array}$ \\
\hline 6.8 Proportion of Protocol Type & $\begin{array}{l}\text { Numerical proportion of a unique packet protocol type ob- } \\
\text { served }\end{array}$ \\
\hline
\end{tabular}

\subsection{PROGRAMMABLE LOGIC CONTROLLER PERFORMANCE}

The PLC simulating the machining stations and communications with the robot controllers requires CPU resources to perform its required functions. Any performance impacts to the PLC operations will be evident through the recorded metrics described below in Table 7. In contrast to the PLC's responsibilities described in Section 2.4, the KPIs in this section are related to the operational performance of the PLC itself, whereas the KPIs in Section 4.1 are related to the operational performance of the manufacturing process.

The TwinCAT TC3 Engineering module (which executes on the PLC) includes two library functions for capturing the metrics listed in Table 7: TC_CpuUsage, and FB_CxProfiler. The metrics from these two functions are recorded once every second while the experiment is running. All data is stored within the PLC until cleared by an operator via the HMI after the data has been downloaded.

Table 7 - PLC Performance Metrics

\begin{tabular}{|l|l|}
\hline \multicolumn{1}{|c|}{ Metric } & \multicolumn{1}{c|}{ Description } \\
\hline 7.1 Task Execution Time & Time required to complete the PLC task \\
\hline 7.2 Minimum Task Execution Time & $\begin{array}{l}\text { Minimum amount of time required to complete the PLC } \\
\text { task }\end{array}$ \\
\hline 7.3 Maximum Task Execution Time & $\begin{array}{l}\text { Maximum amount of time required to complete the PLC } \\
\text { task }\end{array}$ \\
\hline 7.4 CPU Utilization & Amount of PLC CPU usage \\
\hline 7.5 Maximum CPU Utilization & Maximum amount of PLC CPU usage \\
\hline
\end{tabular}

Table 8 - PLC Performance KPI

\begin{tabular}{|ll|c|}
\hline \multicolumn{2}{|c|}{ Key Performance Indicator } & Description \\
\hline 8.1 & Task Execution Time & Mean and standard deviation of PLC task execution time \\
\hline 8.2 & CPU Utilization & Mean and standard deviation of PLC CPU utilization \\
\hline
\end{tabular}

\subsection{SERVER PERFORMANCE}

The servers within the enclave require computing resources (e.g., CPU time, memory usage, disk usage) to perform their tasks during an experiment (e.g., robot control). To capture the performance of these resources, a Python application was created to capture and log the required metrics. All of the metrics are logged in comma-separated values files at a specified rate, which is defined by the researcher through command line arguments (the default rate is one sample per second). 
To perform the capture, the researcher executes the script from the command line interface, along with required arguments, before the experiment is initiated. Once initiated, the script will continue capturing the metrics until it is stopped. The script can also automatically cease its measurements by listening for broadcast messages on the local network that indicate the end of an experiment.

Although there is a separate measurement system capturing the network traffic of the enclave, it is important to note the inclusion of server-based network measurements (Metrics 9.7-9.10). Some of the cybersecurity tools used during experiments block traffic from the wire, before a packet has the chance to reach a server. Measuring at the server allows a comparison of traffic flow on the network to what is actually consumed and generated by a specific server. However, one important difference between the network capture and server performance measurements is the latter does not include raw packet data; only simple flow counters.

Table 9 - Server Performance Metrics

\begin{tabular}{|ll|l|}
\hline \multicolumn{1}{|c|}{ Metric } & \multicolumn{1}{c|}{ Description } \\
\hline 9.1 & CPU Utilization Timers & Amount of server CPU utilization \\
\hline 9.2 Available Memory & Amount of server memory available by the server \\
\hline 9.3 Total Memory & Total amount of memory installed in the server \\
\hline 9.4 Disk I/O Byte Counters & $\begin{array}{l}\text { Number of bytes read and written to the server hard } \\
\text { drive(s) }\end{array}$ \\
\hline 9.5 Disk I/O Access Counters & $\begin{array}{l}\text { Number of discrete read and write operations to the server } \\
\text { hard drive(s) since the last sample }\end{array}$ \\
\hline 9.7 Network Byte Counters & $\begin{array}{l}\text { Amount of time used to read and write to the server hard } \\
\text { drive(s) since the last sample }\end{array}$ \\
\hline 9.8 Network Packet Counters & \begin{tabular}{l} 
Number of bytes transmitted and received by the server \\
\hline 9.9
\end{tabular} Network Packet Errors & $\begin{array}{l}\text { Number of packets transmitted and received by the server } \\
\text { the server }\end{array}$ \\
\hline 9.10 Network Dropped Packets & $\begin{array}{l}\text { Number of incoming or outgoing packets dropped by the } \\
\text { server }\end{array}$ \\
\hline
\end{tabular}

Table 10 - Server Performance KPI

\begin{tabular}{|l|l|}
\hline \multicolumn{1}{|c|}{ Key Performance Indicator } & \multicolumn{1}{c|}{ Description } \\
\hline 10.1 CPU Utilization & Mean and standard deviation of server CPU utilization \\
\hline 10.2 Memory Utilization & Mean and standard deviation of server memory utilization \\
\hline 10.3 Average Disk I/O & $\begin{array}{l}\text { Mean rate and standard deviation of data read and written } \\
\text { to the server hard drive(s) }\end{array}$ \\
\hline 10.4 Network Throughput (Bits) & $\begin{array}{l}\text { Mean rate and standard deviation of bits transmitted and } \\
\text { received by the server }\end{array}$ \\
\hline 10.5 Network Throughput (Packets) & $\begin{array}{l}\text { Mean rate and standard deviation of packets transmitted } \\
\text { and received by the server }\end{array}$ \\
\hline
\end{tabular}


5 APPENDIX A - DESCRIPTION OF MEASUREMENTS AND KPI

\begin{tabular}{|l|l|}
\hline ID & 1.1 \\
\hline Name & Part Timestamps $\left(p_{A}^{x, S}, p_{D}^{x, S}\right)$ \\
\hline Description & Timestamping of unique part events (e.g. arriving and departing stations) \\
\hline Unit of measure & seconds \\
\hline Notes & $\begin{array}{l}\text { Each part has an arrival and departure timestamp for each station, where } \\
p_{A}^{x, S}=\text { the arrival timestamp of part } x \text { as station } S, \text { and } p_{D}^{x, S} \text { is the departure } \\
\text { of timestamp of part } x \text { as station } S . \\
\text { When a part arrives or departs a simulated machining station, the PLC will } \\
\text { log the time. The PLC tracks time as discrete } 10 \text { millisecond time steps from } \\
\text { the start of the production cycle. The timestamps are converted to seconds } \\
\text { for analysis. }\end{array}$ \\
\hline
\end{tabular}

\begin{tabular}{|c|c|}
\hline ID & 1.2 \\
\hline Name & Station Timers $\left(S_{P}^{S}, S_{F}^{S}, S_{I}^{S}\right)$ \\
\hline Description & Accumulation of time a station is in any valid state \\
\hline Unit of measure & seconds \\
\hline Notes & $\begin{array}{l}\text { Simulated machining stations have three operating states: } \\
\text { - Processing - station is loaded and the part is being processed } \\
\text { - } \quad \text { Finished - station is loaded and waiting for the robot to remove the } \\
\text { - } \quad \text { Idle - station is unloaded and waiting for a part to be delivered }\end{array}$ \\
\hline
\end{tabular}




\begin{tabular}{|l|l|}
\hline ID & 1.3 \\
\hline Name & Batch Timer $\left(b_{P}\right)$ \\
\hline Description & Total amount of time required for the batch to be produced \\
\hline Unit of measure & seconds \\
\hline Notes & $\begin{array}{l}\text { The timer is started after the operator puts the enclave into the operational } \\
\text { state, and is stopped when enclave operations end (automatic or operator } \\
\text { shutdown). }\end{array}$ \\
\hline
\end{tabular}

\begin{tabular}{|l|l|}
\hline ID & 1.4 \\
\hline Name & Batch Parts Counter $\left(b_{Q}\right)$ \\
\hline Description & Number of parts produced in the batch \\
\hline Unit of measure & quantity \\
\hline
\end{tabular}

\begin{tabular}{|l|l|}
\hline ID & 2.1 \\
\hline Name & Part Production Time $\left(E_{P}\right)$ \\
\hline Description & Amount of time required for a part to be produced \\
\hline Formula & $\begin{array}{l}\text { where } E_{P}^{x}=p_{D}^{x, S_{n}}-p_{A}^{x, S_{0}} \\
\text { at the last station, } s_{n} ; \text { and } p_{A}^{x, S_{0}}=\text { arrival timestamp of part } x \text { at the first sta- } \\
\text { tion, } s_{0} .\end{array}$ \\
\hline Unit of measure & seconds \\
\hline Notes & $\begin{array}{l}\text { This metric is calculated by the PLC after the part has departed the final sta- } \\
\text { tion. }\end{array}$ \\
\hline
\end{tabular}




\begin{tabular}{|c|c|}
\hline ID & 2.2 \\
\hline Name & Cycle Time $\left(E_{C}\right)$ \\
\hline Description & Amount of time between finished parts \\
\hline Formula & $\begin{array}{l}\qquad E_{C}^{x}=p_{D}^{x, S_{4}}-p_{D}^{x-1, S_{4}} \\
\text { where } E_{C}^{x}=\text { cycle time of part } x ; p_{D}^{x, S_{4}}=\text { departure timestamp of part } x \text { at sta- } \\
\text { tion } s_{4} ; \text { and } p_{D}^{x-1, S_{4}}=\text { departure timestamp of the previous part, } x-1 \text {, at sta- } \\
\text { tion } s_{4} \text {. }\end{array}$ \\
\hline Unit of measure & seconds \\
\hline
\end{tabular}

\begin{tabular}{|c|c|}
\hline ID & 2.3 \\
\hline Name & Throughput Rate $\left(E_{R}\right)$ \\
\hline Description & Number of parts produced over a specific amount of time \\
\hline Formula & $\begin{array}{l}\qquad E_{R}^{T}=\frac{\Delta b_{Q}^{T}}{t_{i}-t_{0}} * 3600 \text { seconds } \\
\text { with } \\
\qquad T=t_{0} \leq t<t_{i} \\
\text { where } E_{R}^{T}=\text { throughput rate during timespan } T \text {; and } \Delta b_{Q}^{T}=\text { change in the } \\
\text { batch part counter during timespan } T \text {. To calculate the throughput of the to- } \\
\text { tal batch, the formula can be rewritten as: } \\
\qquad E_{R}=\frac{b_{Q}}{b_{P}} * 3600 \text { seconds } \\
\text { where } E_{R}=\text { batch throughput rate; } b_{Q}=\text { quantity of parts produced in the } \\
\text { batch; and } b_{P}=\text { total amount of time required to produce the batch }\end{array}$ \\
\hline Unit of measure & parts / hour \\
\hline
\end{tabular}




\begin{tabular}{|c|c|}
\hline ID & 2.4 \\
\hline Name & Production Effectiveness $\left(E_{E}\right)$ \\
\hline Description & $\begin{array}{l}\text { Relationship between the planned production time to complete a batch and } \\
\text { the actual production time }\end{array}$ \\
\hline Formula & $\begin{array}{l}\qquad E_{E}=\frac{\widehat{E_{C}} * b_{Q}}{b_{P}} \\
\text { where } \widehat{E_{P}}=\text { estimated cycle time between finished parts; } b_{Q}=\text { the total num- } \\
\text { ber of parts produced in the batch; and } b_{P}=\text { total amount of time required to } \\
\text { produce the batch. }\end{array}$ \\
\hline Unit of measure & $\%$ \\
\hline Notes & $\begin{array}{l}\text { For the purpose of this experiment, the estimated part production time }\left(\widehat{E_{P}}\right) \\
\text { is calculated by finding the mean cycle time between parts from the baseline } \\
\text { experiments }\left(\overline{E_{P b}}\right) \text { : } \\
\qquad \widehat{E_{C}}=\overline{E_{P b}}=\frac{1}{b_{Q b}} \sum_{x=1}^{b_{Q b}} E_{C b}^{x}, \\
\text { where } b_{Q b}=\text { number of parts produced during the baseline experiments, and } \\
E_{C b}^{x}=\text { cycle time between parts required to produce part } x \text { during the base- } \\
\text { line experiments. }\end{array}$ \\
\hline
\end{tabular}

\begin{tabular}{|c|c|}
\hline ID & 2.5 \\
\hline Name & Station Allocation Ratio $\left(E_{A}\right)$ \\
\hline Description & $\begin{array}{l}\text { Ratio between the amount of time a station was busy and the batch produc- } \\
\text { tion time }\end{array}$ \\
\hline Formula & $\begin{array}{l}\qquad E_{A}^{S}=\frac{S_{P}^{S}+S_{F}^{S}}{b_{P}} \\
\text { where } E_{A}^{S}=\text { allocation ratio of station } S ; s_{P}^{S}+s_{F}^{S}=\text { amount of time station } S \\
\text { was busy; and } b_{P}=\text { total amount of time required to produce the batch. }\end{array}$ \\
\hline Unit of measure & $\%$ \\
\hline Notes & A "busy" station is defined as any station that is not in the "idle" state. \\
\hline
\end{tabular}




\begin{tabular}{|c|c|}
\hline ID & 2.6 \\
\hline Name & Station Utilization Efficiency $\left(E_{U}\right)$ \\
\hline Description & $\begin{array}{l}\text { Ratio between the amount of time a station was producing and the amount } \\
\text { of time the batch required to complete }\end{array}$ \\
\hline Formula & $\begin{array}{l}\qquad E_{U}^{S}=\frac{S_{P}^{S}}{b_{P}} \\
\text { where } E_{U}^{S}=\text { utilization efficiency of station } S ; s_{P}^{S}=\text { amount of time station } S \\
\text { was in the "processing" state; and } b_{P}=\text { total amount of time required to pro- } \\
\text { duce the batch. }\end{array}$ \\
\hline Unit of measure & $\%$ \\
\hline
\end{tabular}

\begin{tabular}{|c|c|c|c|c|c|c|}
\hline ID & \multicolumn{6}{|l|}{3.1} \\
\hline Name & \multicolumn{6}{|c|}{ Joint Position $\left(j_{\theta P}\right)$} \\
\hline Description & \multicolumn{6}{|c|}{ Position of each robot joint } \\
\hline Unit of measure & \multicolumn{6}{|l|}{ radians } \\
\hline \multirow[t]{3}{*}{ Range } & & Joint 1 & Joint 2 & Joint 3 & Joint 4 & Joint 5 \\
\hline & Minimum & 0 & 0 & 0 & 0 & 0 \\
\hline & Maximum & 5.90 & 2.70 & 5.16 & 3.60 & 5.87 \\
\hline Notes & \multicolumn{6}{|c|}{$\begin{array}{l}\text { Joint encoder values are measured relative to the home pose values. The for- } \\
\text { mula used to convert the robot encoder values to radians is: } \\
\qquad j_{\theta}^{h}=\frac{\left|\eta_{m}^{h}\right|}{\eta_{p}} * \lambda^{h} * 2 \pi \\
\text { where } j_{\theta}^{h}=\text { angle in radians of joint } h ; \eta_{m}^{h}=\text { encoder value of joint } h ; \eta_{p}=\text { rated } \\
\text { encoder pulses per revolution; and } \lambda^{h}=\text { gear ratio of joint } h \text {. }\end{array}$} \\
\hline
\end{tabular}




\begin{tabular}{|l|l|}
\hline ID & 3.2 \\
\hline Name & Joint Setpoint $\left(j_{S}\right)$ \\
\hline Description & Control setpoint of each robot joint \\
\hline Unit of measure & radians \\
\hline Range & Minimum 0 \\
\cline { 2 - 3 } & Maximum 5.90 \\
\hline Notes & $\begin{array}{l}\text { Joint encoder values are measured relative to the home pose values. The for- } \\
\text { mula used to convert the robot encoder values to radians is: }\end{array}$ \\
& $\begin{array}{l}\text { where } j_{\theta}^{h}=\text { angle in radians of joint } h ; \eta_{m}^{h}=\text { encoder value of joint } h ; \eta_{p}=\text { rated } \\
\text { encoder pulses per revolution; and } \lambda^{h}=\text { gear ratio of joint } h .\end{array}$ \\
\hline
\end{tabular}

\begin{tabular}{|l|l|}
\hline ID & 3.3 \\
\hline Name & Joint Velocity $\left(j_{\dot{\theta}}\right)$ \\
\hline Description & Velocity of each robot joint \\
\hline Unit of measure & radians per second \\
\hline Notes & The formula used to convert the robot velocity data to radians per second is: \\
& $\begin{array}{l}\text { where } j_{\dot{\theta}}^{h}=\frac{\omega^{h}}{60} * \lambda^{h} * 2 \pi, \quad, \quad \text { velocity of joint } h ; \omega^{h}=\text { revolutions per minute of the motor at } \\
\text { joint } h ; \text { and } \lambda^{h}=\text { the gear ratio of the transmission of joint } h .\end{array}$ \\
\hline
\end{tabular}




\begin{tabular}{|l|l|}
\hline ID & 3.4 \\
\hline Name & Joint Current $\left(j_{I}\right)$ \\
\hline Description & Electrical current of each robot joint \\
\hline Unit of measure & amperes \\
\hline Notes & Electrical current measurements are received from the robot in milliamperes. \\
\hline
\end{tabular}

\begin{tabular}{|l|l|}
\hline ID & 3.5 \\
\hline Name & Joint Control Flags $\left(j_{F}\right)$ \\
\hline Description & Flags describing the operational status of each robot joint \\
\hline Notes & $\begin{array}{l}\text { Flags are included for each joint, and include: communication timeout, initial- } \\
\text { ization complete, position reached, joint controller mode, sensor error, mo- } \\
\text { tor halted, over-temperature, over-voltage, under-voltage, and over-current. }\end{array}$ \\
\hline
\end{tabular}

\begin{tabular}{|l|l|}
\hline ID & 4.1 \\
\hline Name & Actuation Latency $\left(R_{L}\right)$ \\
\hline Description & $\begin{array}{l}\text { Amount of time required for the robot arm to begin movement after a new } \\
\text { job is received }\end{array}$ \\
\hline Formula & $R_{L}^{J}=r_{a}^{P_{0}^{J}}-r_{m}^{J} \quad$, \\
& $\begin{array}{l}\text { where } R_{L}^{J}=\text { the actuation latency for job } J ; r_{a}^{P_{0}^{J}}=\text { timestamp of actuation initi- } \\
\text { ation at pose } P_{0} \text { of job } J ; \text { and } r_{m}^{J}=\text { timestamp of receipt of job } J .\end{array}$ \\
\hline Unit of measure & seconds \\
\hline Notes & $\begin{array}{l}\text { The timestamp of actuation initiation, } r_{a} \text {, is found by identifying when joint 4 } \\
\text { has rotated greater than or equal to } \pm 0.001 \text { radians from pose } P_{0} .\end{array}$ \\
\hline
\end{tabular}




\begin{tabular}{|c|c|}
\hline ID & 4.2 \\
\hline Name & Pose Travel Time $\left(R_{P}\right)$ \\
\hline Description & Amount of time for the robot arm to move between two poses \\
\hline Formula & $\begin{array}{l}\qquad R_{P}^{P_{i \rightarrow j}}=\left\{\begin{array}{c}r_{b}^{P_{j}}-r_{m}^{J} \\
r_{b}^{P_{j}}-r_{a}^{P_{i}}\end{array} \text { if } P_{i}=P_{0}\right. \\
\text { where } R_{P}^{P_{i \rightarrow j}}=\text { travel time required to move between pose } P_{i} \text { and the next } \\
\text { pose } P_{j} ; r_{b}^{P_{j}}=\text { timestamp of actuation completion at pose } P_{j} ; r_{m}^{J}=\text { timestamp } \\
\text { of receipt of job } J ; \text { and } r_{a}^{P_{i}}=\text { timestamp of actuation initiation at pose } P_{i} .\end{array}$ \\
\hline Unit of measure & seconds \\
\hline Notes & $\begin{array}{l}\text { For the initial actuation timestamp of any job }\left(r_{a}^{P_{0}}\right) \text {, the timestamp used is } \\
\text { the job receipt time }\left(r_{m}^{J}\right) \text {. For all other cases, the timestamp of actuation initi- } \\
\text { ation }\left(r_{a}\right) \text { is found by identifying when joint } 4 \text { has rotated greater than or } \\
\text { equal to } \pm 0.001 \text { radians from pose } P_{0} \text {. }\end{array}$ \\
\hline
\end{tabular}

\begin{tabular}{|c|c|}
\hline ID & 4.3 \\
\hline Name & Position Accuracy $\left(R_{A}\right)$ \\
\hline Description & Deviation between the commanded position and the measured position \\
\hline Formula & $\begin{array}{l}\text { where } \\
\qquad R_{A}=\sqrt{\left(\bar{x}-x_{c}\right)^{2}+\left(\bar{y}-y_{c}\right)^{2}+\left(\bar{z}-z_{c}\right)^{2}} \\
\qquad \bar{x}=\frac{1}{n} \sum_{k=1}^{n} x_{k}, \quad \bar{y}=\frac{1}{n} \sum_{k=1}^{n} y_{k}, \quad \bar{z}=\frac{1}{n} \sum_{k=1}^{n} z_{k} \\
\text { where } x_{c}, y_{c} \text {, and } z_{c}=\text { coordinates of the commanded pose; } x_{k}, y_{k} \text {, and } z_{k}= \\
\text { coordinates of the } k \text {-th attained pose; and } n=\text { number of measurement sam- } \\
\text { ples. }\end{array}$ \\
\hline Unit of measure & millimeters \\
\hline
\end{tabular}




\begin{tabular}{|c|c|}
\hline ID & 4.4 \\
\hline Name & Position Repeatability $\left(R_{R}\right)$ \\
\hline Description & $\begin{array}{l}\text { Closeness of agreement between the measured position after } n \text { repeat visits } \\
\text { to the same commanded position }\end{array}$ \\
\hline Formula & 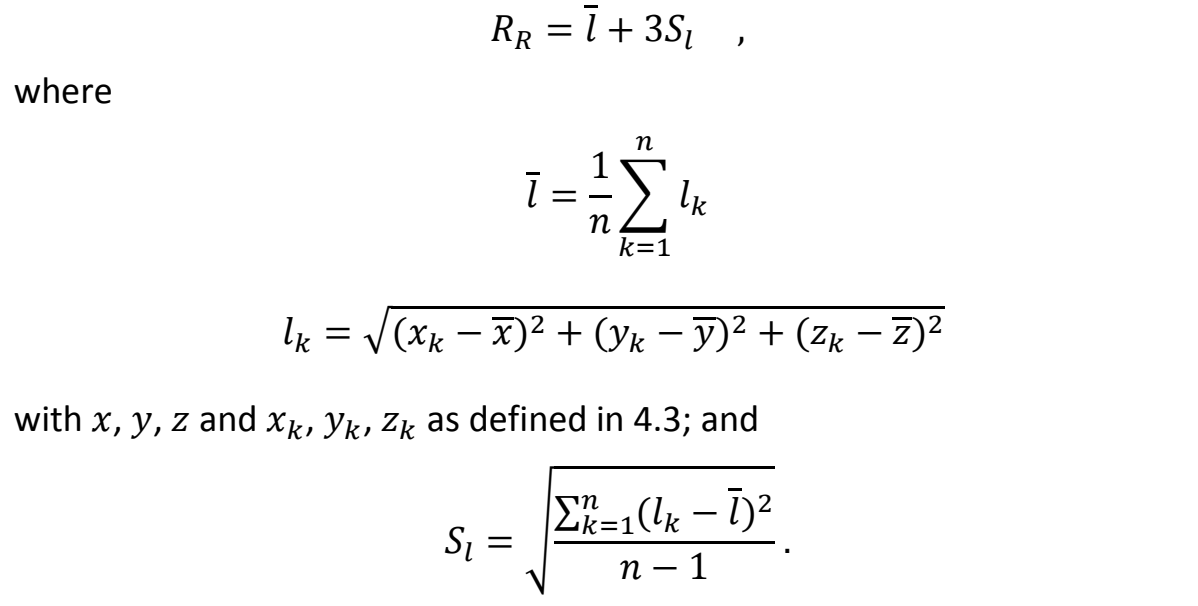 \\
\hline Unit of measure & millimeters \\
\hline
\end{tabular}

\begin{tabular}{|c|c|}
\hline ID & 4.5 \\
\hline Name & Energy Consumption $\left(R_{E}\right)$ \\
\hline Description & Estimation of the amount of power consumed by the robot joint motors \\
\hline Formula & $\begin{array}{l}\qquad R_{E}^{T}=\sum_{h=1}^{H} \int_{t_{0}}^{t_{i}} j_{I}^{h}(t)^{2} \Omega_{h} d t \\
\text { with } \\
\qquad T=t_{0} \leq t<t_{i} \\
\text { where } R_{E}^{T}=\text { energy consumption during the timespan } T ; H=\text { total number of } \\
\text { joints; } t_{0}=\text { initial sample time; } t_{i}=\text { final sample time; } j_{I}^{h}(t)^{2}=\text { square of the } \\
\text { current at joint } h \text { at time } t ; \Omega_{h}=\text { resistance of the motor terminal at joint } h .\end{array}$ \\
\hline Unit of measure & joules \\
\hline Notes & $\begin{array}{l}\text { Terminal resistance } \Omega_{j} \text { of the joint motors } 1 \text { through } 5 \text { are defined for each } \\
\text { joint as: } 0.978 \Omega, 0.978 \Omega, 0.978 \Omega, 4.48 \Omega, 13.7 \Omega \text {, respectively. }\end{array}$ \\
\hline
\end{tabular}




\begin{tabular}{|c|c|}
\hline ID & 4.6 \\
\hline Name & Job Execution Time $\left(R_{T}\right)$ \\
\hline Description & Amount of time required for a robot to complete a job \\
\hline Formula & $\begin{array}{l}\qquad R_{T}^{J}=r_{b}^{P_{\text {final }}^{J}}-r_{m}^{J} \\
\text { where } R_{T}^{J}=\text { the execution time for job } J ; r_{b}^{P_{\text {final }}^{J}}=\text { timestamp of actuation } \\
\text { completion of the final pose } P \text { of job } J \text {; and } r_{m}^{J}=\text { timestamp of receipt of } \\
\text { job } J \text {. }\end{array}$ \\
\hline Unit of measure & seconds \\
\hline
\end{tabular}

\begin{tabular}{|l|l|}
\hline ID & 5.1 \\
\hline Name & Packet Headers $\left(n_{H}\right)$ \\
\hline Description & Header data of a captured packet \\
\hline Notes & $\begin{array}{l}\text { Headers may include appended data from the tools used to capture the } \\
\text { packet. }\end{array}$ \\
\hline
\end{tabular}

\begin{tabular}{|l|l|}
\hline ID & 5.2 \\
\hline Name & Packet Data $\left(n_{D}\right)$ \\
\hline Description & Application layer payload of a captured packet \\
\hline Notes & $\begin{array}{l}\text { Allows monitoring of multiple subsystems within the enclave, and enables in- } \\
\text { spection of the enclave operations across multiple nodes. }\end{array}$ \\
\hline
\end{tabular}

\begin{tabular}{|l|l|}
\hline ID & 5.3 \\
\hline Name & Packet Protocol Type $\left(n_{K}\right)$ \\
\hline Description & Protocol type of a captured packet \\
\hline Notes & $\begin{array}{l}\text { Protocol types of captured packets may change based on the applied cyber- } \\
\text { security technologies for each individual experiment. }\end{array}$ \\
\hline
\end{tabular}




\begin{tabular}{|l|l|}
\hline ID & 5.4 \\
\hline Name & Packet Timestamp $\left(n_{T}\right)$ \\
\hline Description & Timestamp of a captured packet \\
\hline Unit of measure & seconds \\
\hline Notes & $\begin{array}{l}\text { Post-processed timestamps have their epoch converted to the start time of } \\
\text { the experiment. Raw data timestamps from an enclave experiment are meas- } \\
\text { ured relatively from the Unix epoch. }\end{array}$ \\
\hline
\end{tabular}

\begin{tabular}{|c|c|c|}
\hline ID & \multicolumn{2}{|l|}{5.5} \\
\hline Name & \multicolumn{2}{|c|}{ Packet Size $\left(n_{S}\right)$} \\
\hline Description & \multicolumn{2}{|c|}{ Size of a packet in bytes } \\
\hline Unit of measure & \multicolumn{2}{|l|}{ bytes } \\
\hline \multirow[t]{2}{*}{ Range } & Minimum & 0 \\
\hline & Maximum & 65535 \\
\hline
\end{tabular}

\begin{tabular}{|l|l|}
\hline ID & 5.6 \\
\hline Name & Packet Errors Counter $\left(n_{E}\right)$ \\
\hline Description & Number of packets transmitted or received with errors \\
\hline
\end{tabular}

\begin{tabular}{|l|l|}
\hline ID & 5.7 \\
\hline Name & Dropped Packets Counter $\left(n_{D}\right)$ \\
\hline Description & Number of packets dropped by the network \\
\hline
\end{tabular}




\begin{tabular}{|l|l|}
\hline ID & 5.8 \\
\hline Name & Packet Counter $\left(n_{C}\right)$ \\
\hline Description & Number of packets transmitted and received \\
\hline Notes & $\begin{array}{l}\text { Measurement may be in reference to enclave, between nodes, or to/from a } \\
\text { single node. }\end{array}$ \\
\hline
\end{tabular}

\begin{tabular}{|c|c|}
\hline ID & 6.1 \\
\hline Name & Packet Path Delay $\left(N_{D}\right)$ \\
\hline Description & Time delay along the path from transmitter to receiver \\
\hline Formula & $\begin{array}{l}\qquad N_{D}^{p, a \rightarrow b}=n_{T}^{p, b}-n_{T}^{p, a} \\
\text { where } N_{D}^{p, i \rightarrow j}=\text { path delay for packet } p \text { from node } i \text { to } j ; n_{T}^{p, b}=\text { timestamp of } \\
\text { packet } p \text { when received at node } b ; n_{T}^{p, j}=\text { timestamp of packet } p \text { when trans- } \\
\text { mitted at node } a \text {. }\end{array}$ \\
\hline Unit of measure & seconds \\
\hline Notes & $\begin{array}{l}\text { Path delay calculations are especially useful when cybersecurity hardware is } \\
\text { added to the wire, allowing accurate measurement of any network perfor- } \\
\text { mance impacts. }\end{array}$ \\
\hline
\end{tabular}

\begin{tabular}{|c|c|}
\hline ID & 6.2 \\
\hline Name & Inter-packet Delay $\left(N_{J}\right)$ \\
\hline Description & Difference between the packet path delay of two packets \\
\hline Formula & $\begin{array}{l}\qquad N_{J}^{p_{i}}=N_{D}^{p_{i}}-N_{D}^{p_{i-1}} \\
\text { where } N_{J}^{p_{i}}=\text { time delay between packet } p_{i} \text { and the previous packet; } N_{D}^{p_{i}}= \\
\text { packet path delay for packet } p_{i} ; \text { and } N_{D}^{p_{i-1}}=\text { packet path delay for packet } \\
p_{i-1} \text {. }\end{array}$ \\
\hline Unit of measure & seconds \\
\hline Notes & $\begin{array}{l}\text { When using this KPI to described the inter-packet delay variation for a series } \\
\text { of packets, the result is typically communicated by minimum and maximum } \\
\text { values, mean and standard deviation, or a histogram. }\end{array}$ \\
\hline
\end{tabular}




\begin{tabular}{|l|l|}
\hline ID & 6.3 \\
\hline Name & TCP Packet Round Trip Time $\left(N_{R}\right)$ \\
\hline Description & $\begin{array}{l}\text { Amount of time for the source node of a packet to receive the acknowledge- } \\
\text { ment of receipt (ACK) from the destination node }\end{array}$ \\
\hline Formula & $\begin{array}{l}\text { where } n_{T}^{p}=n_{T}^{a}-n_{T}^{p} \\
\text { received ACK for packet } p .\end{array}$ \\
\hline Unit of measure & seconds \\
\hline Notes & This KPI is also known as round-trip time (RTT). \\
\hline
\end{tabular}

\begin{tabular}{|c|c|c|}
\hline ID & \multicolumn{2}{|l|}{6.4} \\
\hline Name & \multicolumn{2}{|c|}{ Information Ratio $\left(N_{I}\right)$} \\
\hline Description & \multicolumn{2}{|c|}{ Ratio of the quantity of process information packets to all packets } \\
\hline Formula & \multicolumn{2}{|c|}{$\begin{array}{l}\qquad N_{I}=\frac{n_{I}}{n_{C}} \\
\text { where } n_{I}=\text { number of information packets. }\end{array}$} \\
\hline Unit of measure & \multicolumn{2}{|l|}{$\%$} \\
\hline \multirow[t]{2}{*}{ Range } & Minimum & 0 \\
\hline & Maximum & 100 \\
\hline Notes & \multicolumn{2}{|c|}{$\begin{array}{l}\text { Information packets are defined as: any packet containing information that i } \\
\text { used to operate the manufacturing process. }\end{array}$} \\
\hline
\end{tabular}




\begin{tabular}{|c|c|}
\hline ID & 6.5 \\
\hline Name & Bit Rate $\left(N_{B}\right)$ \\
\hline Description & Rate of bits transmitted or received over a specific timespan \\
\hline Formula & $\begin{array}{l}\qquad \overline{N_{B}}=\frac{8}{t_{i}-t_{0}} \sum_{t \in T} n_{S}^{t} \\
\text { with } \\
\qquad T=\left\{x \in \mathbb{S}: t_{0}<x \leq t_{i}\right\} \\
\text { where } n_{S}^{t}=\text { the size of the packet at time } t \text { in bytes; } \mathbb{S}=\text { set of captured pack- } \\
\text { ets; and } t_{0}, t_{i}=\text { initial and final time, respectively, of the desired timespan. }\end{array}$ \\
\hline Unit of measure & bits / second \\
\hline Notes & $\begin{array}{l}\text { Specific derivatives of the KPI may be calculated as the average bits per sec- } \\
\text { ond over the total experiment time, or calculated over discrete intervals. }\end{array}$ \\
\hline
\end{tabular}

\begin{tabular}{|l|l|}
\hline ID & 6.6 \\
\hline Name & Packet rate $\left(N_{P}\right)$ \\
\hline Description & Rate of packets transmitted and received over a specific amount of time \\
\hline Formula & $\overline{N_{P}}=\frac{n_{C}^{t_{i}}-n_{C}^{t_{0}}}{t_{i}-t_{0}} \quad$, \\
& $\begin{array}{l}\text { where } n_{C}^{t_{i}}=\text { number of packets sent or received at time } t_{i} ; n_{C}^{t_{0}}=\text { number of } \\
\text { onds; and } t_{0}=\text { measurement period start time in seconds. }\end{array}$ \\
\hline Unit of measure & packets / second \\
\hline Notes & $\begin{array}{l}\text { Specific derivatives of the KPI may be calculated as the average packets per } \\
\text { second over the total experiment time, or calculated over discrete intervals. }\end{array}$ \\
\hline
\end{tabular}




\begin{tabular}{|c|c|}
\hline ID & 6.7 \\
\hline Name & Packet Error Rate $\left(N_{E}\right)$ \\
\hline Description & $\begin{array}{l}\text { Rate of packets transmitted or received with errors over a specific amount of } \\
\text { time }\end{array}$ \\
\hline Formula & $\begin{array}{l}\qquad \bar{N}_{E}=\frac{n_{E}^{t_{i}}-n_{E}^{t_{0}}}{t_{i}-t_{0}} \\
\text { where } n_{E}^{t_{i}}=\text { number of packets sent or received with errors at time } t_{i} ; n_{E}^{t_{0}}= \\
\text { number of packets sent or received with errors at time } t_{0} ; t_{i}=\text { measurement } \\
\text { period end time in seconds; and } t_{0}=\text { measurement period start time in sec- } \\
\text { onds. }\end{array}$ \\
\hline Unit of measure & packets / second \\
\hline Notes & $\begin{array}{l}\text { Specific derivatives of the KPI may be calculated as the average number of } \\
\text { packets with errors per second over the total experiment time, or calculated } \\
\text { over discrete intervals. }\end{array}$ \\
\hline
\end{tabular}

\begin{tabular}{|c|c|c|}
\hline ID & \multicolumn{2}{|l|}{6.8} \\
\hline Name & \multicolumn{2}{|c|}{ Proportion of Protocol Type $\left(N_{T}\right)$} \\
\hline Description & \multicolumn{2}{|c|}{ Numerical proportion of a unique packet protocol type observed } \\
\hline Unit of measure & \multicolumn{2}{|l|}{$\%$} \\
\hline \multirow{2}{*}{ Range } & Minimum & 0 \\
\hline & Maximum & 100 \\
\hline Notes & \multicolumn{2}{|c|}{$\begin{array}{l}\text { Captured packet protocol types included in this KPI may change based on the } \\
\text { applied cybersecurity technologies for each individual experiment. }\end{array}$} \\
\hline
\end{tabular}




\begin{tabular}{|l|l|}
\hline ID & 7.1 \\
\hline Name & Task Execution Time $\left(m_{E}\right)$ \\
\hline Description & Time required to complete the PLC task \\
\hline Unit of measure & seconds \\
\hline Notes & $\begin{array}{l}\text { PLC task execution time data is recorded as the average task execution time } \\
\text { of each cycle over a one second period. }\end{array}$ \\
\hline
\end{tabular}

\begin{tabular}{|l|l|}
\hline ID & 7.2 \\
\hline Name & Minimum Task Execution Time $\left(m_{F}\right)$ \\
\hline Description & Minimum amount of time required to complete the PLC task \\
\hline Unit of measure & seconds \\
\hline
\end{tabular}

\begin{tabular}{|l|l|}
\hline ID & 7.3 \\
\hline Name & Maximum Task Execution Time $\left(m_{G}\right)$ \\
\hline Description & Maximum amount of time required to complete the PLC task \\
\hline Unit of measure & seconds \\
\hline
\end{tabular}

\begin{tabular}{|c|c|c|}
\hline ID & \multicolumn{2}{|l|}{7.4} \\
\hline Name & \multicolumn{2}{|c|}{ CPU Utilization $\left(m_{T}\right)$} \\
\hline Description & \multicolumn{2}{|c|}{ Amount of PLC CPU usage } \\
\hline Unit of measure & \multicolumn{2}{|l|}{$\%$} \\
\hline \multirow[t]{2}{*}{ Range } & Minimum & 0 \\
\hline & Maximum & 100 \\
\hline Notes & \multicolumn{2}{|c|}{ PLC CPU utilization data is recorded by the PLC every 100 milliseconds. } \\
\hline
\end{tabular}




\begin{tabular}{|c|c|c|}
\hline ID & \multicolumn{2}{|l|}{7.5} \\
\hline Name & \multicolumn{2}{|c|}{ Maximum CPU Utilization $\left(m_{V}\right)$} \\
\hline Description & \multicolumn{2}{|c|}{ Maximum amount of PLC CPU usage } \\
\hline Unit of measure & \multicolumn{2}{|l|}{$\%$} \\
\hline \multirow[t]{2}{*}{ Range } & Minimum & 0 \\
\hline & Maximum & 100 \\
\hline Notes & \multicolumn{2}{|c|}{ PLC CPU utilization data is recorded by the PLC every 100 milliseconds. } \\
\hline
\end{tabular}

\begin{tabular}{|l|l|}
\hline ID & 8.1 \\
\hline Name & Task Execution Time Distribution $\left(M_{E}\right)$ \\
\hline Description & Mean and standard deviation of PLC task execution time \\
\hline Formula & \multicolumn{1}{|c|}{$M_{E}=\overline{m_{E}} \pm \sigma_{m_{E}}$} \\
\hline Unit of measure & Seconds \\
\hline
\end{tabular}

\begin{tabular}{|l|l|}
\hline ID & 8.2 \\
\hline Name & CPU Utilization Distribution $\left(M_{T}\right)$ \\
\hline Description & Mean and standard deviation of PLC CPU utilization \\
\hline Formula & \multicolumn{1}{c|}{$M_{T}=\overline{m_{T}} \pm \sigma_{m_{T}}$} \\
\hline Unit of measure & $\%$ \\
\hline
\end{tabular}




\begin{tabular}{|c|c|}
\hline ID & 9.1 \\
\hline Name & CPU Utilization Timers $\left(c_{U}\right)$ \\
\hline Description & Amount of server CPU utilization \\
\hline Unit of measure & seconds \\
\hline Notes & $\begin{array}{l}\text { Metric is recorded as the total amount of utilization seconds across all CPUs } \\
\text { within the server [8], [9]. The CPU utilization modes recorded for the metric } \\
\text { are: } \\
\text { - } \quad \text { user - amount of time the CPU was executing non-kernel processes } \\
\text { - } \quad \text { idle - amount of time the CPU had nothing to process } \\
\text { - } \quad \text { iowait - amount of time the CPU was waiting for an I/O operation to } \\
\text { - } \text { complete (e.g. hard drive read or write) } \\
\text { - } \quad \text { softirq - amount of time the CPU was servicing a software interrupt } \\
\text { - } \quad \text { steal - amount of time a virtualized CPU was waiting to use the CPU } \\
\text { - guest - amount of time the CPU spent executing processes for a } \\
\text { - gosted virtual machine } \\
\text { - } \text { cess for a hosted virtual machine (see nice) } \\
\text { nice - amount of time the CPU spent executing a process with a } \\
\text { The medified execution priority (can be either a higher or lower priority) } \\
\text { script. For example, the system CPU time is denoted as } c_{U_{\text {system }} \text {. }}\end{array}$ \\
\hline
\end{tabular}




\begin{tabular}{|l|l|}
\hline ID & 9.2 \\
\hline Name & Available Memory $\left(c_{A}\right)$ \\
\hline Description & Amount of server memory available by the server \\
\hline Unit of measure & bytes \\
\hline
\end{tabular}

\begin{tabular}{|l|l|}
\hline ID & 9.3 \\
\hline Name & Total Memory $\left(c_{M}\right)$ \\
\hline Description & Total amount of memory installed in the server \\
\hline Unit of measure & bytes \\
\hline Notes & This metric is measured once at the beginning of the experiment. \\
\hline
\end{tabular}

\begin{tabular}{|l|l|}
\hline ID & 9.4 \\
\hline Name & Disk I/O Byte Counters $\left(c_{F}\right)$ \\
\hline Description & Number of bytes read and written to the server hard drive $(\mathrm{s})$ \\
\hline Unit of measure & bytes \\
\hline Notes & $\begin{array}{l}\text { Each measurement has a read and write component: } \\
C_{F}=\left(c_{F R}, c_{F W}\right)\end{array}$, \\
& $\begin{array}{l}\text { where the subscript }(\mathrm{R} \text { or } \mathrm{W}) \text { defines whether the metric describes disk reads } \\
\text { or writes. }\end{array}$ \\
\hline
\end{tabular}




\begin{tabular}{|l|l|}
\hline ID & 9.5 \\
\hline Name & Disk I/O Counters $\left(c_{G}\right)$ \\
\hline Description & $\begin{array}{l}\text { Number of discrete read and write operations to the server hard drive }(\mathrm{s}) \\
\text { since the last sample }\end{array}$ \\
\hline Notes & $\begin{array}{l}\text { Each measurement has a read and write component: } \\
c_{G}=\left(c_{G R}, c_{G W}\right) \quad, \quad \\
\text { where the subscript }(\mathrm{R} \text { or } \mathrm{W}) \text { defines whether the metric describes disk reads } \\
\text { or writes. }\end{array}$ \\
\hline
\end{tabular}

\begin{tabular}{|l|l|}
\hline ID & 9.6 \\
\hline Name & Disk I/O Access Time $\left(c_{H}\right)$ \\
\hline Description & $\begin{array}{l}\text { Amount of time used to read and write to the server hard drive }(s) \text { since the } \\
\text { last sample }\end{array}$ \\
\hline Unit of measure & seconds \\
\hline Notes & $\begin{array}{l}\text { Each measurement has a read and write component: } \\
C_{H}=\left(c_{H R}, c_{H W}\right),\end{array}$ \\
& where the subscript (R or W) defines disk reads or writes, respectively. \\
\hline
\end{tabular}

\begin{tabular}{|l|l|}
\hline ID & 9.7 \\
\hline Name & Network Byte Counters $\left(c_{B}\right)$ \\
\hline Description & Number of bytes transmitted and received by the server \\
\hline Unit of measure & bytes \\
\hline Notes & $\begin{array}{l}\text { Each measurement has a transmit and receive component: } \\
C_{B}=\left(c_{B T}, C_{B R}\right)\end{array}$, \\
& $\begin{array}{l}\text { where the subscript }(\mathrm{T} \text { or R) defines network transmission or receipt, respec- } \\
\text { tively. }\end{array}$ \\
\hline
\end{tabular}




\begin{tabular}{|l|l|}
\hline ID & 9.8 \\
\hline Name & Network Packet Counters $\left(c_{P}\right)$ \\
\hline Description & Number of packets transmitted and received by the server \\
\hline Unit of measure & bytes \\
\hline Notes & $\begin{array}{l}\text { Each measurement has a transmit and receive component: } \\
C_{P}=\left(C_{P T}, C_{P R}\right)\end{array}$, \\
& \begin{tabular}{l} 
where the subscript (T or R) defines network transmission or receipt, respec- \\
\hline
\end{tabular}
\end{tabular}

\begin{tabular}{|l|l|}
\hline ID & 9.9 \\
\hline Name & Network Packet Errors $\left(c_{E}\right)$ \\
\hline Description & Number of packets with errors transmitted and received by the server \\
\hline Notes & $\begin{array}{l}\text { Each measurement has a transmit and receive component: } \\
C_{E}=\left(c_{E T}, c_{E R}\right)\end{array}$, \\
$\begin{array}{l}\text { where the subscript }(\mathrm{T} \text { or R) defines network transmission or receipt, respec- } \\
\text { tively. }\end{array}$ \\
\hline
\end{tabular}

\begin{tabular}{|l|l|}
\hline ID & 9.10 \\
\hline Name & Network Dropped Packets $\left(c_{X}\right)$ \\
\hline Description & Number of incoming or outgoing packets dropped by the server \\
\hline Notes & $\begin{array}{l}\text { Each measurement has a transmit and receive component: } \\
c_{X}=\left(c_{X T}, c_{X R}\right)\end{array}$ \\
& $\begin{array}{l}\text { where the subscript }(T \text { or R) defines network transmission or receipt, respec- } \\
\text { tively. }\end{array}$ \\
\hline
\end{tabular}




\begin{tabular}{|c|c|c|}
\hline ID & \multicolumn{2}{|l|}{10.1} \\
\hline Name & \multicolumn{2}{|c|}{ CPU Utilization $\left(C_{U}\right)$} \\
\hline Description & \multicolumn{2}{|c|}{ Mean and standard deviation of server CPU utilization } \\
\hline Formula & \multicolumn{2}{|c|}{ 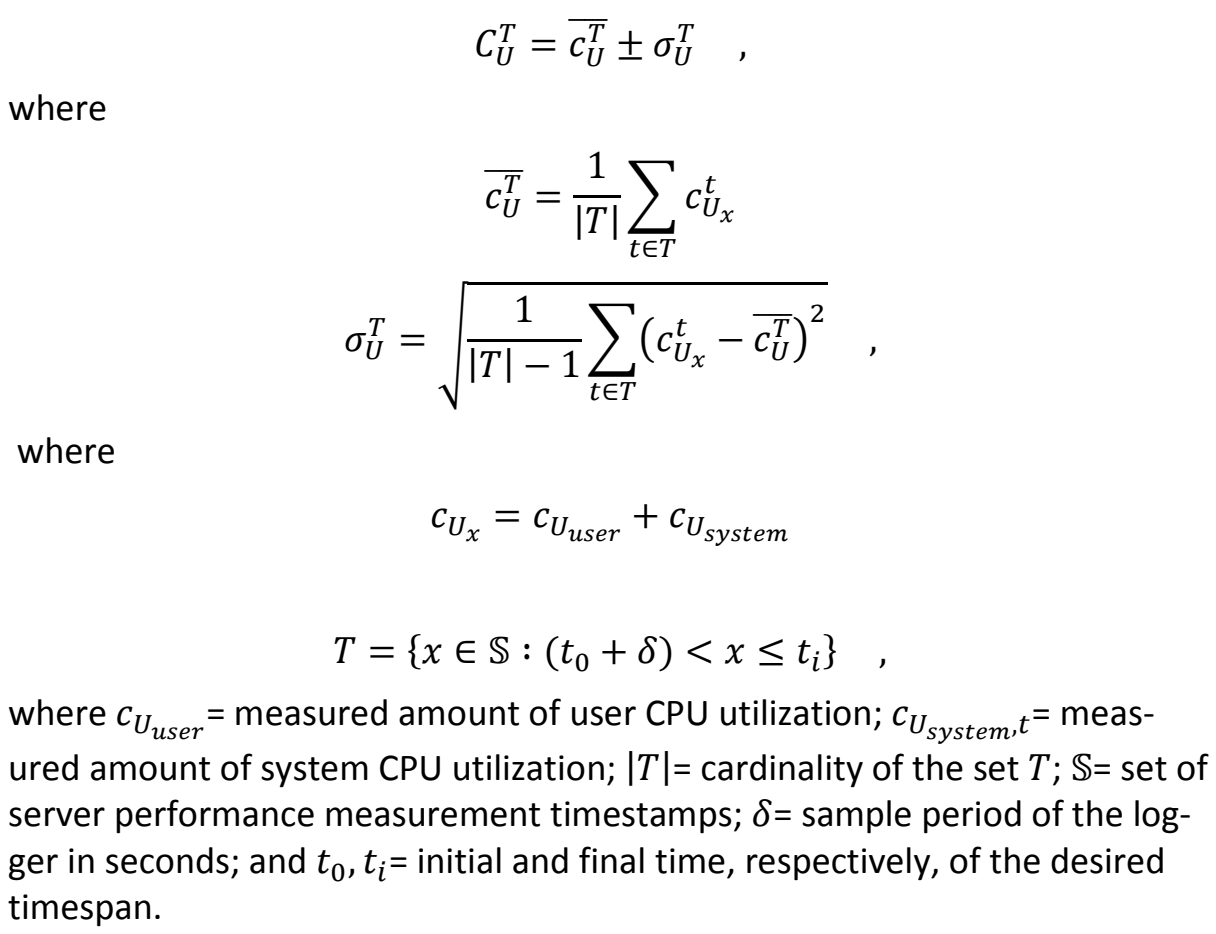 } \\
\hline Unit of measure & \multicolumn{2}{|l|}{$\%$} \\
\hline \multirow[t]{2}{*}{ Range } & Minimum & 0 \\
\hline & Maximum & 100 \\
\hline Notes & \multicolumn{2}{|c|}{$\begin{array}{l}\text { Specific derivatives of the KPI may be calculated over the total experiment } \\
\text { time, or calculated between specific time intervals. } \\
\text { The set } T \text { does not include the nearest sample } t_{0} \text { because the resulting calcu- } \\
\text { lation would include data that are outside of the desired timespan. To ac- } \\
\text { count for this, the sample period of the logger }(\delta) \text { is used to coerce the set to } \\
\text { only include samples that have measured data within the specified timespan. }\end{array}$} \\
\hline
\end{tabular}




\begin{tabular}{|c|c|c|}
\hline ID & \multicolumn{2}{|l|}{10.2} \\
\hline Name & \multicolumn{2}{|c|}{ Memory Utilization $\left(C_{X}\right)$} \\
\hline Description & \multicolumn{2}{|c|}{ Mean and standard deviation of server memory utilization } \\
\hline Formula & \multicolumn{2}{|c|}{$\begin{array}{l}\text { where } \\
\qquad \begin{array}{c}C_{X}^{T}=\overline{c_{X}^{T}} \pm \sigma_{X}^{T} \\
c_{X}^{T}=\frac{1}{|T|} \sum_{t \in T} c_{X}^{t}\end{array} \\
\qquad \sigma_{X}^{T}=\sqrt{\frac{1}{|T|-1} \sum_{t \in T}\left(c_{X}^{t}-\overline{c_{X}^{T}}\right)^{2}}, \\
\text { where } \\
\qquad T=\left\{x \in \mathbb{S}: t_{0} \leq x \leq t_{i}\right\} \\
\text { where } c_{X, t}=\text { amount of utilized server memory at time } t ; c_{M}=\text { total amount of } \\
\text { server memory; } c_{A, t}=\text { total amount of available memory at time } t ;|T|=\text { cardi- } \\
\text { nality of the set } T ; \mathbb{S}=\text { set of server performance measurement timestamps; } \\
\text { and } t_{0}, t_{i}=\text { initial and final time, respectively, of the desired timespan. }\end{array}$} \\
\hline Unit of measure & \multicolumn{2}{|l|}{$\%$} \\
\hline \multirow[t]{2}{*}{ Range } & Minimum & 0 \\
\hline & Maximum & 100 \\
\hline Notes & \multicolumn{2}{|c|}{$\begin{array}{l}\text { Specific derivatives of the KPI may be calculated over the total experiment } \\
\text { time, or calculated between specific time intervals. }\end{array}$} \\
\hline
\end{tabular}




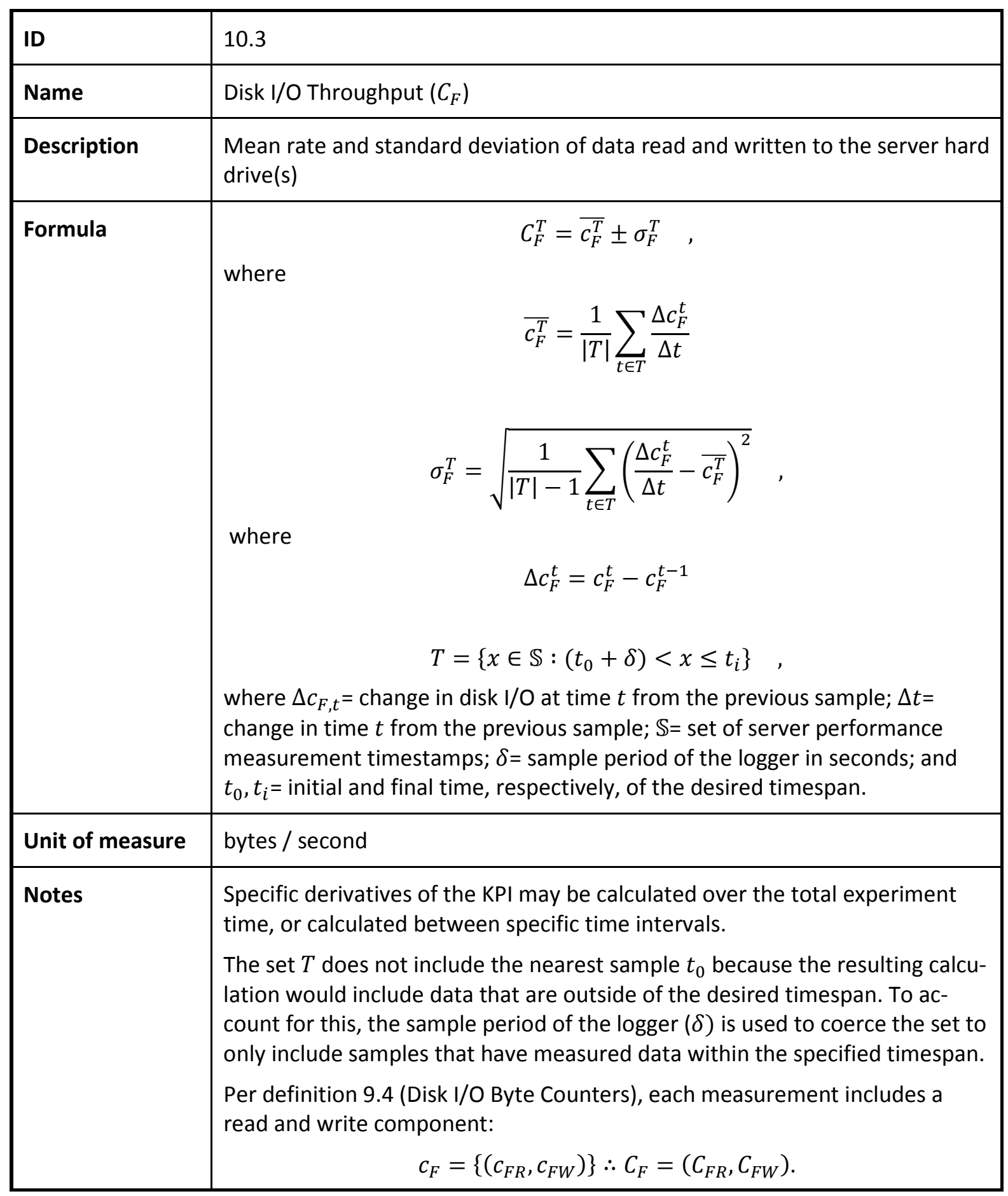




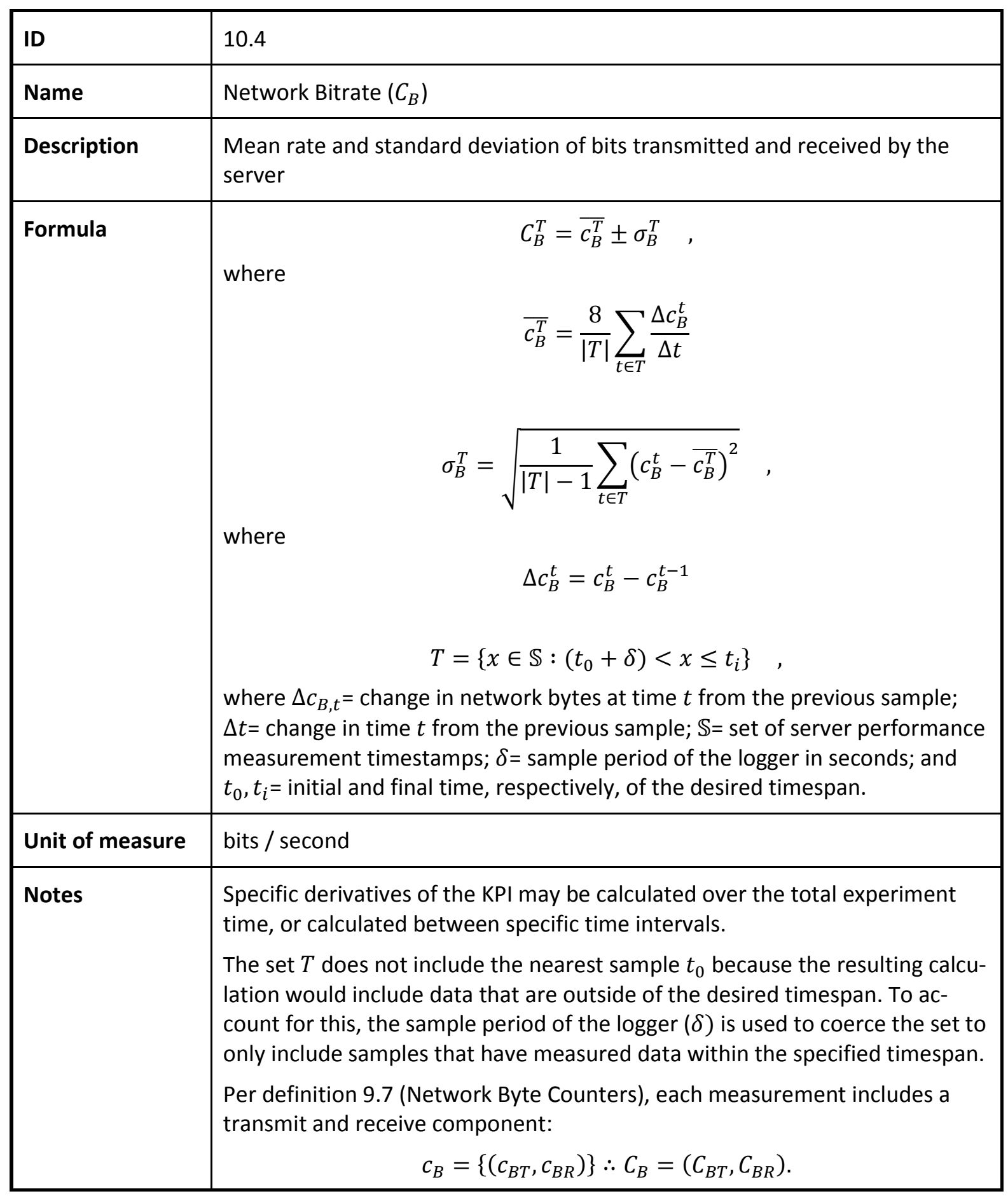




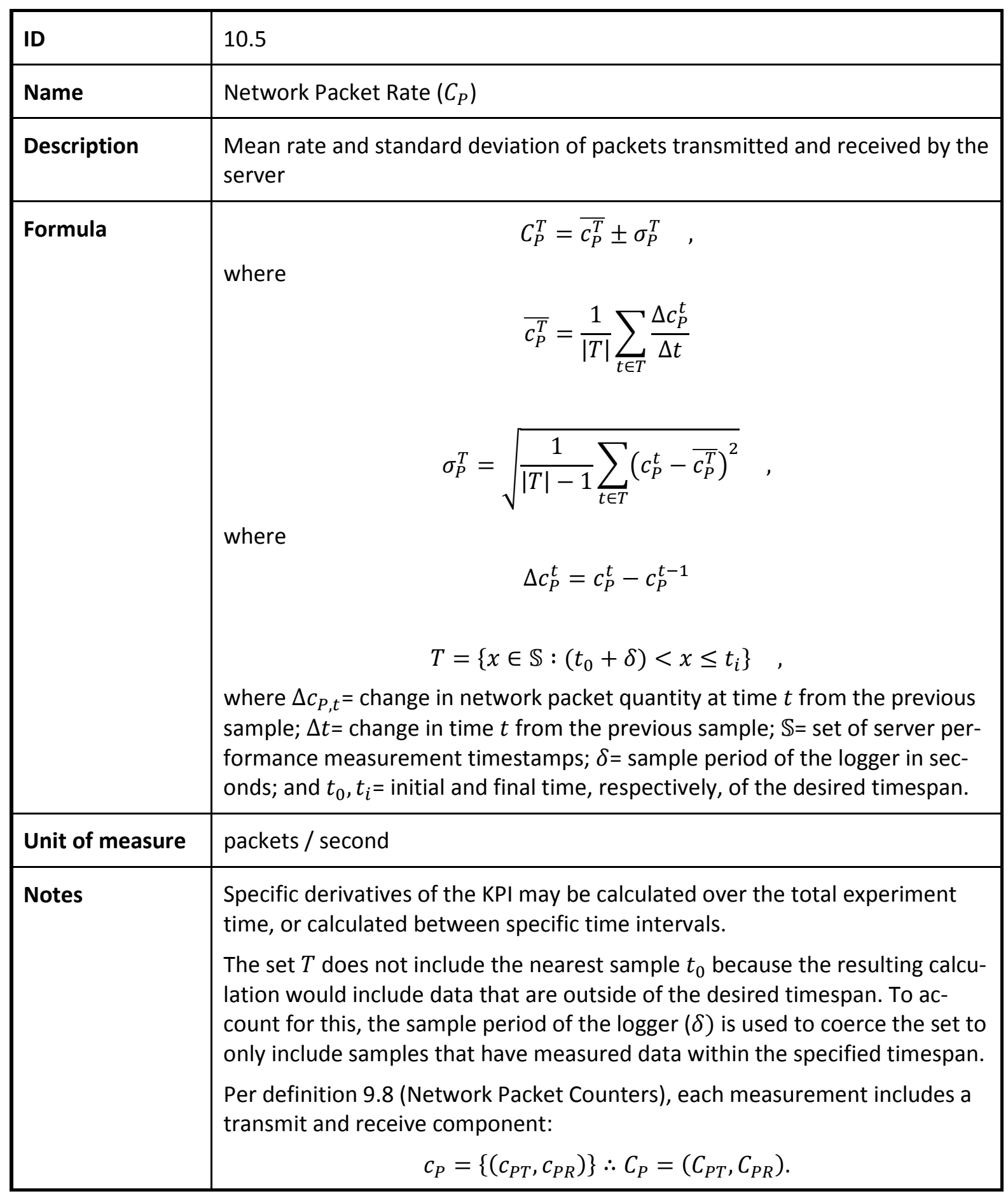


6 APPENDIX B - REFERENCES

[1] R. Candell, T. Zimmerman, and K. Stouffer, "An Industrial Control System Cybersecurity Performance Testbed," NISTIR 8089, National Institute of Standards and Technology (NIST), 2015.

[2] I. C. Garretson, M. Mani, S. Leong, K. W. Lyons, and K. R. Haapala, "Terminology to support manufacturing process characterization and assessment for sustainable production," J. Clean. Prod., vol. 139, pp. 986-1000, 2016.

[3] "Manipulating industrial robots - Performance criteria and related test methods." ISO Standard 9283, 1998.

[4] "Automation systems and integration - Key performance indicators (KPIs) for manufacturing operations management." ISO Standard 22400, 2014.

[5] R. Baroudi, KPI Mega Library: 17,000 Key Performance Indicators. Scotts Valley, California: CreateSpace Independent Publishing Platform, 2010.

[6] C. Demichelis and P. Chimento, "IP Packet Delay Variation Metric for IP Performance Metrics (IPPM), RFC 3393," Internet Eng. Task Force, 2002.

[7] H. Allen and C. Vincente, "Network Performance Definitions and Analysis," in PacNOG 5, 2009.

[8] G. Rodola', "psutil documentation -- psutil 5.1.2 documentation," pythonhosted.org, 2017. [Online]. Available: http://pythonhosted.org/psutil/. [Accessed: 09-Feb-2017].

[9] D. Haynes, "Understanding Linux CPU Stats," ScoutApp.com, 2015. [Online]. Available: http://blog.scoutapp.com/articles/2015/02/24/understanding-linuxs-cpu-stats. [Accessed: 09-Feb-2017]. 